Working Paper 06-53

Economics Series 20

August 2006
Departamento de Economía

Universidad Carlos III de Madrid

Calle Madrid, 126

28903 Getafe (Spain)

Fax (34) 916249875

\title{
PURE STRATEGY EQUILIBRIA OF SINGLE AND DOUBLE AUCTIONS WITH INTERDEPENDENT VALUES
}

Aloisio Araujo ${ }^{1}$ and Luciano I. de Castro ${ }^{2}$

\begin{abstract}
We prove the existence of monotonic pure strategy equilibrium for many types of asymmetric auctions with $n$ bidders and unitary demands, interdependent values and independent types. The assumptions require monotonicity only in the own bidder's type. The payments can be a function of all bids. Thus, we provide a new equilibrium existence result for asymmetrical double auctions and a small number of bidders. The generality of our setting requires the use of special tie-breaking rules. We present a reasonable counterexample for interdependent values auctions that shows that sometimes all equilibria are trivial, that is, they have zero probability of trade. Nevertheless, we give sufficient conditions for non-trivial equilibrium existence.
\end{abstract}

JEL Classification Numbers: C62, C72, D44, D82.

Keywords: equilibrium existence in auctions, pure strategy Nash equilibrium, monotonic equilibrium, tie-breaking rule.

Corresponding author: decastro.luciano@gmail.com. We are grateful to the participants of the 2005 Decentralization Conference and Econometric Society World Congress and to Paulo K. Monteiro, Humberto Moreira and two anonymous referees for very helpful suggestions.

\footnotetext{
${ }^{1}$ Aloisio Araujo: Getulio Vargas Foundation and IMPA

${ }^{2}$ Luciano I. de Castro: Universidad Carlos III de Madrid
} 


\section{Introduction}

Since the first works on asymmetrical auctions, by Vickrey (1961) and Griesmer, Levitan and Shubik (1967), many theoretical papers have considered the question of the equilibrium existence for such games, among which we can cite Amman and Leininger (1996), Lebrun (1999), Lizzeri and Persico (2000), Maskin and Riley (2000), Athey (2001), Reny and Zamir (2004) and Jackson and Swinkels (2005). ${ }^{1}$ The methods to prove the existence of equilibrium are essentially of two kinds. The first papers appeal to a system of differential equations whose solution is shown to be an equilibrium. The later ones discretize the space of types or bids, obtain the equilibrium in this case and then prove that the limit when the grid is made fine is an equilibrium. ${ }^{2}$

In this paper, we take another route to prove the equilibrium existence for monotonic asymmetrical auctions with independent types. ${ }^{3}$ We allow assumptions weaker than the usual: we do not assume that the utilities are increasing in all types but only in the own bidder's type, and payment can depend on all bids. Thus, we treat in a single framework many kinds of asymmetrical auctions with unitary demand, including double auctions.

We work on the set of non-decreasing functions, $N$, and of smooth increasing functions, $I$. We prove that the set of best responses to functions in $I$ is a "unitary" subset of $N .{ }^{4}$ Thus, convexity of the set of best replies is straightforward. We make a perturbation of the original auction to avoid the problem of discontinuities. Since $N$ is compact in the $\mathcal{L}^{1}$ topology, we can use the Kakutani-Fan-Glicksberg Theorem to obtain easily a fixed point. The limit of these fixed points is shown to be an equilibrium under an appropriate tie-breaking rule.

We prove equilibrium existence with an endogenous tie-breaking rule à la Simon and Zame (1990) and Jackson, Simon, Swinkels and Zame (2002), henceforth JSSZ. As discussed in subsection 3.1, the need of a special tie-breaking rule is unavoidable in our setting.

This is the first equilibrium existence result for asymmetric double auctions with interdependent values and a small number of players (see section 5 for a discussion on the literature). Thus, we are also interested in establishing that the equilibrium is not trivial, that is, there is a positive probability of trade. This was established for private value auctions by Jackson and Swinkels (2001) under quite general conditions. Nevertheless, since they are restricted to private values, their proof does not directly apply. Moreover, for interdependent value auctions, it is possible to give examples where trade occurs with zero probability even when there is a positive probability of the values of buyers being above the values of sellers (see example

\footnotetext{
${ }^{1}$ While Vickrey (1961), Athey (2001) and Jackson and Swinkels (2005) consider many kinds of games and Amman and Leininger (1996) treat all-pay auctions, the rest of these papers are mainly concerned with first-price auctions, as are Griesmer, Levitan and Shubik (1967). Vickrey (1961) analyzes mainly symmetric auctions.

${ }^{2}$ Jackson and Swinkels (2005) can be considered an exception, because they appeal to more general theorems about the existence of Nash equilibrium.

${ }^{3}$ Our method is related to Fundenberg, Mobius and Szeil (2003). See section 5.

${ }^{4}$ This needs a qualification. It is not exactly unitary. See Remark 1.
} 
3). This is related to Akerlof's (1970) 'market for lemons' example on the effects of private information. Thus, additional assumptions are needed. We make such assumptions and obtain the result about the positive probability of trade.

The connection between Akerlof's market for lemons and the problem of nontrade in auctions with interdependent values should not be surprising. Indeed, auctions provide a good setup for analyzing general equilibrium models with asymmetric information. More than that, as Milgrom (1981) argues, auction theory is useful to explain or solve some paradox of general equilibrium theory, such as that related to rational expectations. Although Milgrom (1981) is able to provide explanations of such paradoxes, his models are restricted to second price auctions. Since double auctions are more realistic models of the reality, one can view our results as a contribution to Milgrom's program of establishing strategic foundation for rational expectations equilibrium through auction theory.

The paper is organized as follows. In section 2 we describe the model. In section 3 we present our equilibrium existence result and a useful auxiliary result. In section 4 we give sufficient conditions for double auctions having a non-trivial equilibrium, that is, an equilibrium with a positive probability of trade. The conclusion (section 5 ) is a discussion about the contributions of the paper and the related literature. An appendix collects the proofs.

\section{The Model}

Let $P=\{1, \ldots, n\}$ be the set of players. Players are sellers, each possessing one object, or buyers, who are interested in buying just one object. The game may consist of only buyers or only sellers, in which case we say that the auction is singlesided. It is convenient to say that $P=S \cup B$, where players $j \in S$ are sellers and players $i \in B$ are buyers.

Some of these players can be non-strategic, in which case the utility functions defined below do not exist. To asssume the existence of non-strategic players is useful in single-sided auctions or to prove the existence of trade in double auctions. For instance, we can model a single-sided auction with $n_{B}$ buyers and $n_{o}$ objects by assuming the existence of $n_{o}$ non-strategic sellers so that there are $n=n_{o}+n_{B}$ players. Non-strategic but secret reserve prices can also be modeled as bids of non-strategic sellers. Although non-strategic players do not have a relevant payoff function, they will be treated exactly as strategic players (with signals and covert bids), but with an exogenously fixed strategy. This does not introduce any problem and makes the statements easier. Nevertheless, the reader should keep in mind that Assumptions 2-8 below, about the utility functions, refer only to strategic players.

Player $i \in\{1, \ldots, n\}$ receives a unidimensional private information, $t_{i}$, and chooses an action that is a real number (i.e., she submits a bid $b_{i}$ ). The auctioneer compares the bids and determines who receives one object and who does not, according to an allocation rule specified below.

If player $i$ receives one object, her payoff is $\bar{u}_{i}(t, b)$ and if she receives no object, her payoff is $\underline{u}_{i}(t, b)$, where $t=\left(t_{i}, t_{-i}\right)$ is the profile of all signals and $b=\left(b_{i}, b_{-i}\right)$ is the profile of bids. The dependence on all bids and the asymmetry of the functions 
allow modeling all players in double auctions and exotic single-sided auctions, where the payment is a function of all bids.

2.1. Information. We assume independence of types. Because $\bar{u}_{i}(t, b)$ and $\underline{u}_{i}(t, b)$ can have any form, we may assume without loss of generality that the private signal of each player, $t_{i}$, is a real number uniformly distributed in $[0,1] .^{5}$ To summarize, we require the following:

Assumption 1. Types are independent and uniformly distributed on $[0,1]$.

The main role of Assumption 1 is to obtain the existence of a monotonic best reply, when the opponents follow strictly increasing strategies, which is the theme of our first result (Theorem 1 below). This states the existence and (a qualified) uniqueness of the best reply. Although we use independence to simplify arguments in general, it seems possible to modify all other proofs to accommodate dependence (in the case where there exists a strictly positive density function on $[0,1]^{n}$ ), provided we have the consequences pointed by Theorem 1. Unfortunately, even under affiliation, the conclusion of Theorem 1 is not always true (see Reny and Zamir, 2004 for a counter-example). Because of this, we maintain such an assumption throughout the paper.

2.2. Bidding. After receiving the private information, each player submits a sealed proposal, that is, a bid $b_{i} \in \mathbb{R}$. There is a reserve price $\underline{b} \geq 0$ and a maximum valid bid $\bar{b}$, which are commonly known. ${ }^{6}$ In addition, buyers can take a nonparticipation decision, $b_{O U T}^{B}<\underline{b}$, which ensures them the payoff of zero. In the same way, sellers can take a non-participation decision $b_{O U T}^{S}>\bar{b}$. Thus, the action space is $\left\{b_{O U T}^{B}\right\} \cup[\underline{b}, \bar{b}]$ for buyers and $[\underline{b}, \bar{b}] \cup\left\{b_{O U T}^{S}\right\}$ for sellers. For simplicity, we will make an abuse of notation by defining

$$
\mathcal{B} \equiv\left\{b_{O U T}^{B}\right\} \cup[\underline{b}, \bar{b}] \cup\left\{b_{O U T}^{S}\right\}
$$

as the action space. As said earlier, $b$ stands for the profiles of bids, that is, $b=\left(b_{1}\right.$, $\left.b_{2}, \ldots, b_{n}\right) \in \mathcal{B}^{n}$. As usual, we will use the notation $b_{-i}=\left(b_{1}, \ldots, b_{i-1}, b_{i+1}, \ldots, b_{n}\right)$ and write $b=\left(b_{i}, b_{-i}\right)$.

\footnotetext{
${ }^{5}$ Assume that the original type is $h_{i}$, distributed on $\left[\underline{h}_{i}, \bar{h}_{i}\right]$ according to the strictly increasing and continuous c.d.f. $F_{i}(\cdot)$ and that the value of the object is given by $v_{i}\left(h_{i}, h_{-i}\right)$. Then, we can define $t_{i}=F_{i}\left(h_{i}\right)$ and $u_{i}\left(t_{i}, t_{-i}\right) \equiv v_{i}\left(F_{i}^{-1}\left(t_{i}\right), F_{-i}^{-1}\left(t_{-i}\right)\right)$. Now, the type $t_{i}$ is uniformly distributed on $[0,1]$. Thus, our assumption rules out just the cases of atoms or gaps in the distribution of types.

${ }^{6}$ If there is no reserve price (in the usual sense), let $\underline{b}=0$. We are assuming a maximum permitted bid to rule out behaviors (equilibria) in which one bidder bids arbitrarily high and the others bid zero. This could happen in third price auctions, for instance.
} 
2.3. Allocation. The allocation of the objects is done according to an allocation rule (or function) $a: \mathcal{B}^{n} \rightarrow[0,1]^{n}$, with the meaning that, given a profile of bids $b \in \mathcal{B}^{n}$, if $a_{i}(b)=1$ then player $i$ receives one object at the end of the auction, that is, if $i$ is a buyer, she buys one object, whereas if $i$ is a seller, she keeps her object. We allow $a_{i}(b)$ to take values between 0 and 1 in order to model the situation where a player receives the object with a probability between 0 and 1 . For instance, when there is a tie, it is usual to split the object randomly among the tying bidders. (We will call this as the standard tie-breaking rule).

JSSZ point out that some auctions do not have equilibrium with the standard tiebreaking rule (see their example 1 and also Jackson et. al. 2004). Thus, some special tie-breaking rules are required for a general equilibrium existence result and they may require that the bidders take further actions in the case of a tie. For instance, Maskin and Riley (2000) use a second price auction tie-breaking rule, which requires the bidders involved in a tie to bid in a second-price auction. JSSZ propose that the action is the announcement of their types. To model this, we will assume that the allocation rule is actually defined as a function $a: \mathcal{B}^{n} \times \mathcal{S}^{n} \rightarrow[0,1]^{n}$, where $\mathcal{S}$ is a set of actions used to break ties. We will omit $\mathcal{S}$ when no action is required to break ties. Thus, we have the following:

Definition 1. Let $n_{o}$ be the number of objects in the auction. ${ }^{7}$ A function $a$ : $\mathcal{B}^{n} \times \mathcal{S}^{n} \rightarrow[0,1]^{n}$ is an allocation rule if:

(i) $t \mapsto a(\mathbf{b}(t), \mathbf{s}(t))$ is measurable for all profiles of measurable strategies $(\mathbf{b}, \mathbf{s}) .^{8}$

(ii) For all $(b, s) \in \mathcal{B}^{n} \times \mathcal{S}^{n}, \sum_{i \in P} a_{i}(b, s)=n_{o}$.

(iii) For each $i \in P, a_{i}(b, s)$ is non-decreasing in $b_{i}$ and non-increasing in $b_{j}$, for $j \neq i$.

(iv) For each $i \in P, a_{i}(b, s)$ does not depend on $s$, unless $\sharp\left\{j: b_{j}>b_{i}\right\}<n_{o}$ and $\sharp\left\{j: b_{j}<b_{i}\right\}<n-n_{o}$, that is, unless there is a relevant tie at $b_{i} \cdot{ }^{9}$ $s .^{10}$

(v) For each $i \in P, a_{i}\left(b_{O U T}^{B}, b_{-i}, s\right)=0$ and $a_{i}\left(b_{O U T}^{S}, b_{-i}, s\right)=1$ for all $b_{-i}$ and

Condition (i) is just a technical condition, while condition (ii) establishes that all the objects are received by some bidder (including, possibly, the non-strategic ones). Condition (iii) establishes that an increasing in own bid does not diminish the possibility of winning (and may increase it). Condition (iv) is the restriction that the actions $s$ are important only in cases of ties. Condition (v) is just the formalization of the definition of $b_{O U T}^{B}$ and $b_{O U T}^{S}$.

\footnotetext{
${ }^{7}$ Formally, the number of objects in the auction $n_{o}$ is equal to the number of strategic and non-strategic sellers, $n_{S}$. We introduce the notation $n_{o}$ only to avoid confusion in the case of single-sided auctions (of buyers), where there are no sellers (besides the non-strategic ones).

${ }^{8}$ We will always work with allocation rules that are monotonic in all arguments. Thus, measurability will not be a issue.

${ }^{9} \mathrm{~A}$ tie is relevant only if it brings doubt as to who gets the object. For instance, a tie between the two highest bids is not relevant in an auction with three objects.

${ }^{10}$ Although $b_{O U T}^{B}$ is available only for buyers and $b_{O U T}^{S}$ is available only for sellers, it is convenient to define $a_{i}$ for all $b_{i} \in \mathcal{B}$.
} 
The reader should note that the definition does not require that the bidder with the highest bid receives the object, as occurs in fair auctions. This freedom in the definition will be useful in the statement of our results, but we are interested in fair allocation rules, where each player receives the object if her bid is above $n-n_{o}$ bids. Indeed, consider the following:

Definition 2. Let $n_{o}$ be as above. A function $a: \mathcal{B}^{n} \times \mathcal{S}^{n} \rightarrow[0,1]^{n}$ is a fair allocation rule (or a tie-breaking rule) if it satisfies the properties $(i)-(v)$ above and, additionally:

(vi) $a_{i}(b, s)=1$ if $\sharp\left\{j: b_{j}<b_{i}\right\} \geq n-n_{o}$.

(vii) $a_{i}(b, s)=0$ if $\sharp\left\{j: b_{j}>b_{i}\right\} \geq n_{o}$.

It is easy to see that fair allocation rules define a threshold bid, $T B\left(b_{-i}\right)$, for each player and profile of the opponents' bids, $b_{-i}$, which is just the $n_{o}$-order statistics of the opponents' bids. That is, for each player $i \in P$ and each profile $b_{-i} \in \mathcal{B}^{n-1}$, there is $T B\left(b_{-i}\right) \in \mathcal{B}$ such that $a_{i}(b, s)=1$ if $b_{i}>T B\left(b_{-i}\right)$ and $a_{i}(b, s)=0$ if $b_{i}<T B\left(b_{-i}\right)$. The value of $a_{i}(b, s)$ is unspecified only if $b_{i}=T B\left(b_{-i}\right)$. Condition (iii) implies that $T B: \mathcal{B}^{n-1} \rightarrow \mathcal{B}$ is a non-decreasing function. Thus, conditions (vi) and (vii) above could also be written as:

(vi) $a_{i}(b, s)=1$ if $b_{i}>T B\left(b_{-i}\right)$.

(vii) $a_{i}(b, s)=0$ if $b_{i}<T B\left(b_{-i}\right)$.

The reader should note that the payments are specified in the utility functions $\bar{u}_{i}, \underline{u}_{i}$. Thus, we define an auction as a profile $\mathcal{A}=\mathcal{A}^{a}=\left(P,\left\{\bar{u}_{i}, \underline{u}_{i}\right\}_{i \in P}, n_{o}, \mathcal{B}, \mathcal{S}\right.$, $a$ ). Now, observe that if we let $a$ vary in the set of fair allocation rules, the outcome of the auction is completely defined, except in situations where there are relevant ties. Thus, it is useful to have the following:

Definition 3. An auction with unspecified allocation rule is a profile $\mathcal{A}=(P$, $\left.\left\{\bar{u}_{i}, \underline{u}_{i}\right\}_{i \in P}, n_{o}, \mathcal{B}\right)$. A specification of $\mathcal{A}$ is a profile $\mathcal{A}^{a}=\left(P,\left\{\bar{u}_{i}, \underline{u}_{i}\right\}_{i \in P}, n_{o}, \mathcal{B}, \mathcal{S}\right.$, a), where $a$ is an allocation rule. If we restrict the specification of $\mathcal{A}$ to the set of fair allocation rules, we may say that $\mathcal{A}$ is a fair auction with unspecified tie-breaking rule.

Definition 4. The standard allocation rule or the standard tie-breaking rule is a fair allocation rule where $\mathcal{S}=\{\varnothing\}$ and the ties are broken by giving the object with the same probability to all tying bidders. The standard tie-breaking rule will be denoted by $\bar{a}$. A fair allocation rule (tie-breaking rule) is said to be special if it is not the standard one.

2.4. Assumptions on the payoff functions. Before the formal statement, given below, we summarize our assumptions on the payoff functions $\bar{u}_{i}$ and $\underline{u}_{i}$ : they are required to be continuous (Assumption 2); the net benefit $u_{i} \equiv \bar{u}_{i}-\underline{u}_{i}$ is increasing with the own player's type, but not necessarily in the other players' types (Assumption 3); the utilities are supermodular (Assumption 4) and weakly monotonic on the bids (Assumption 5).

We formalize and discuss each of these assumptions in the sequel: 
Assumption 2. For all $i \in P, \bar{u}_{i}(t, b)$ and $\underline{u}_{i}(t, b)$ are absolutely continuous in $t \in[0,1]^{n}$ and $b \in \mathcal{B}^{n}$.

It is standard in auction theory to assume continuity or differentiability of the utility functions. Assumption 2 weakens differentiability, but rules out continuous functions with singular parts, that is, functions whose image of zero measure sets may have positive measure. Since the domains are compact sets, this implies that the functions are bounded. Absolute continuity implies the existence of derivatives almost everywhere and that the function is equal to the integral of its derivative.

Assumption 3. For all $i \in P, u_{i}(t, b) \equiv \bar{u}_{i}(t, b)-\underline{u}_{i}(t, b)$ is strictly increasing in $t_{i}$.

This is a monotonicity condition weaker than what is usually required. In interdependent value auctions, it is almost always assumed that the functions are increasing in the own bidder's type and non-decreasing in the other types. In contrast, Assumption 3 allows the utility function to be decreasing in the opponents' types. For instance, the example 1 of JSSZ is included in our framework, where $\bar{u}_{i}(b, t)=5+t_{i}-4 t_{-i}-b_{i}$ and $\underline{u}_{i}(b, t)=0$. We will return to this example later on, when we discuss the need of the tie-breaking rule (see Example 2 in subsection 3.1).

Assumption 4. For all $i \in P, \partial_{b_{i}} \bar{u}_{i}(t, b)$ and $\partial_{b_{i}} \underline{u}_{i}(t, b)$ are non-decreasing in $t_{i}$ (where these derivatives exist).

This assumption is only the requirement that $\bar{u}_{i}$ and $\underline{u}_{i}$ be supermodular as functions of $\left(t_{i}, b_{i}\right)$. Indeed, in the case of twice differentiable functions, supermodularity is equivalent to $\partial_{b_{i} t_{i}}^{2} \bar{u}_{i} \geqslant 0$ and $\partial_{b_{i} t_{i}}^{2} \underline{u}_{i} \geqslant 0$. (In the general case with real variables, a function $f(a, x)$ is supermodular if $a^{\prime} \geqslant a, x^{\prime} \geqslant x$ imply $f\left(a^{\prime}, x^{\prime}\right)+f(a, x) \geqslant$ $\left.f\left(a^{\prime}, x\right)+f\left(a, x^{\prime}\right)\right)$.

To see that this assumption is not restrictive in the usual auctions, assume that we have differentiability and consider the second price auctions. In this case, $\bar{u}_{i}(t, b)=$ $U_{i}\left(v_{i}(t)-\max _{j \neq i} b_{i}\right)$ and $\underline{u}_{i}(t, b)=0$. Thus, $\partial_{b_{i} t_{i}}^{2} \bar{u}_{i}=\partial_{b_{i} t_{i}}^{2} \underline{u}_{i}=0$. For the first price auction, $\bar{u}_{i}\left(t_{i}^{\prime}, t_{-i}, b\right)=U_{i}\left(v_{i}(t)-b_{i}\right)$, then $\partial_{b_{i} t_{i}}^{2} \bar{u}_{i}=U_{i}^{\prime \prime} \cdot(-1) \cdot v_{i}^{\prime}$. If $v_{i}^{\prime} \geqslant 0$, as usual, then $\partial_{b_{i} t_{i}}^{2} \bar{u}_{i} \geqslant 0 \Leftrightarrow U_{i}^{\prime \prime} \leqslant 0$, i.e., in this setting, super modularity is equivalent to weak risk aversion. We illustrate below that it is also not restrictive for double auctions (see Lemma 1). Supermodularity is an assumption usually required to prove existence of pure strategy equilibria in auctions (see, for instance, Lizzeri and Persico (2000), Maskin and Riley (2000), Athey (2001), etc.) In this sense, Assumption 4 is a standard one.

Assumptions 1-4 are used in the proof of Theorem 1, which shows, under an additional condition, that if a bid is a best reply for a fixed type, no bid below it will be optimal for a higher type, that is, all best replies are non-decreasing (see Remark 1 for a more precise statement). Our equilibrium existence result (Theorem 2 ) is also valid under Assumptions 1-4. Nevertheless, we consider an additional assumption:

Assumption 5. $\underline{u}_{i}$ and $\bar{u}_{i}$ are both non-decreasing or both non-increasing in $b_{i}$ for all $i \in P$. 
The purpose of this assumption is to offer a direct and simple proof of equilibrium existence. Indeed, Assumption 5 is used only in the proof of Lemma 3, which is valid without it (see footnote 14). The role of this assumption is to obtain convexity of the best-reply correspondence, after the consequences of Theorem 1 . The proof of this fact is direct and elementary and does not require any previous results based on partial order or lattice theory. The simplicity of the proof may be useful for pedagogical purposes and to clarify the issue of convexity for this kind of game.

It should be noted that auctions usually considered in the literature, including double auctions, satisfy Assumption 5, as we show below.

2.5. Examples. This setting applies to a broad class of discontinuous games. For example, $\bar{u}_{i}(t, b)=v_{i}(t)-b_{i}$ and $\underline{u}_{i}(t, b)=0$ correspond to a first price auction with risk neutrality. Thus, $\bar{u}_{i}$ is decreasing in $b_{i}$ and $\underline{u}_{i}$ is constant with it, which implies Assumption 5. If $\bar{u}_{i}(t, b)=v_{i}(t)-b_{i}$ and $\underline{u}_{i}(t, b)=-b_{i}$ we have the all-pay auction and both $\bar{u}_{i}$ and $\underline{u}_{i}$ are decreasing in $b_{i}$, also satisfying Assumption 5 . The same is true if $\bar{u}_{i}(t, b)=v_{i}(t)-\max _{j \neq i} b_{j}$ and $\underline{u}_{i}(t, b)=-b_{i}$, which is called the war of attrition. As pointed out by Lizzeri and Persico (2000), we can also have combinations of these games. For example, $\bar{u}_{i}(t, b)=v_{i}(t)-\alpha b_{i}-(1-\alpha) \max _{j \neq i} b_{j}$ and $\underline{u}_{i}(t, b)=0$, with $\alpha \in(0,1)$, gives a combination of first and second price auctions. Another possibility is the "third price auction" or an auction where the payment is a general function of the others' bids.

The assumptions are also natural to double auctions. Indeed, consider the following specification, which we use in Theorem 3, on the existence of trade with positive probability:

Assumption 6. Let $\bar{u}_{i}(t, b)$ and $\underline{u}_{i}(t, b)$ be defined as follows:

$$
\bar{u}_{i}(t, b)= \begin{cases}U_{i}\left(v_{i}(t)-e_{i}\right), & \text { if } i \in S \\ U_{i}\left(v_{i}(t)-p_{i}(b)-e_{i}\right), & \text { if } i \in B\end{cases}
$$

and

$$
\underline{u}_{i}(t, b)= \begin{cases}U_{i}\left(p_{i}(b)-e_{i}\right), & \text { if } i \in S \\ U_{i}\left(-e_{i}\right), & \text { if } i \in B\end{cases}
$$

where $e_{i} \geq 0$ is a participation fee, which is zero if $b_{i} \in\left\{b_{O U T}^{B}, b_{\text {OUT }}^{S}\right\}$ and $p_{i}(b) \geq 0$ $i s$ the price paid (received) by buyer $i \in B$ (seller $i \in S$ ). Moreover: ( $i$ ) $U_{i}$ is strictly increasing and differentiable for all $i$, with $U_{i}(0)=0$; (ii) $U_{i}$ is concave for all $i \in B$; (iii) $p_{i}(b)$ is differentiable and non-decreasing in $b_{i}$ for all $i$; (iv) $v_{i}$ is strictly increasing in $t_{i}$ for all $i$.

We have the following:

Lemma 1. Assumption 6 implies Assumptions 2-5.

Proof. It is immediate to check Assumptions 2 and 3. For sellers, Assumption 4 holds trivially because $\partial_{b_{i}} \bar{u}_{i}$ and $\partial_{b_{i}} \underline{u}_{i}$ do not depend on $t_{i}$. For buyers, $\partial_{b_{i}} \underline{u}_{i}$ also do not depend on $t_{i}$. Now, $t_{i} \leqslant t_{i}^{\prime}$ implies $v_{i}\left(t_{i}, t_{-i}\right)-p(b) \leqslant v_{i}\left(t_{i}^{\prime}, t_{-i}\right)-p(b)$ by (iv), which in turn implies $-U_{i}^{\prime}\left(v_{i}\left(t_{i}, t_{-i}\right)-p(b)-e_{i}\right) \leqslant-U_{i}^{\prime}\left(v_{i}\left(t_{i}, t_{-i}\right)-p(b)-e_{i}\right)$ because of (i) and (ii). Now, (iii) implies that $\partial_{b_{i}} p(b) \geqslant 0$ and Assumption 4 follows from 
the expression $\partial_{b_{i}} \bar{u}_{i}(b, t)=-U_{i}^{\prime}\left(v_{i}(t)-p(b)\right) \cdot \partial_{b_{i}} p(b)$. Finally, (iii) implies that Assumption 5 is also satisfied.

It should be said that our model is not restricted to auctions. For instance, Bertrand pricing games can also be treated. In a Bertrand pricing game, the firm's payoff is given by $\left(p_{i}-c_{i}(t)\right) D(p)$ if its price $p_{i}$ is below the price of the others and zero otherwise. Here, $D(\cdot)$ stands for the demand and $c_{i}$ is the cost of firm $i$. Thus, we put $\bar{u}_{i}(t, p)=0$ and $\underline{u}_{i}(t, p)=\left(p_{i}-c_{i}(t)\right) D(p)$. Assumptions 2-5 are satisfied if $c_{i}(\cdot)$ and $D(\cdot)$ are absolutely continuous, $c_{i}(\cdot)$ is decreasing with the type $t_{i}$ and $D(\cdot)$ is non-increasing in $p_{i}$, which are natural assumptions to require.

2.6. Notation. Let $\tilde{N}$ be the set of non-decreasing functions from $[0,1]$ to $\mathcal{B}$. For a function $\mathbf{g} \in \tilde{N}$, let $[\mathbf{g}]$ be the equivalence class of the functions that differ of $\mathbf{g}$ only in a set of zero measure. Now, define $N$ as $\{[\mathbf{g}]: \mathbf{g} \in \tilde{N}\}$. The equivalence classes will be referred to as functions, in the usual abuse of terminology. We endow $N$ with the norm topology of $\mathcal{L}^{1}([0,1], \mathbb{R})$. It is easy to see that $N$ is compact and convex. $^{11}$

In order to avoid confusion with the bids, we will use bold letters to denote bidding functions, i.e., $\mathbf{b}=\left(\mathbf{b}_{1}, \ldots, \mathbf{b}_{n}\right) \in N^{n}$. If we fix the strategies of $i$ 's opponents, $\mathbf{b}_{-i}=$ $\left(\mathbf{b}_{1}, \ldots, \mathbf{b}_{i-1}, \mathbf{b}_{i+1}, \ldots \mathbf{b}_{n}\right) \in N^{n-1}$, let

$$
F_{\mathbf{b}_{-i}}(\beta) \equiv \operatorname{Pr}\left(\left\{t_{-i}: a_{i}\left(\beta, \mathbf{b}_{-i}\left(t_{-i}\right)\right)>0\right\}\right) .
$$

Note that $F_{\mathbf{b}_{-i}}$ is a c.d.f. by the properties of $a_{i}$ required by Definition 1 .

If $F_{\mathbf{b}_{-i}}$ is absolutely continuous with respect to the Lebesgue measure, let $f_{\mathbf{b}_{-i}}(\cdot)$ be its Radon-Nykodim derivative. Let us define $b_{*} \equiv \operatorname{ess} \inf \left\{\beta \in[\underline{b}, \bar{b}]: f_{\mathbf{b}_{-i}}(\beta)>0\right\}$ and $b^{*} \equiv \operatorname{ess} \sup \left\{\beta \in[\underline{b}, \bar{b}]: f_{\mathbf{b}_{-i}}(\beta)>0\right\}$. Note that $b_{*}$ and $b^{*}$ vary with $\mathbf{b}_{-i}$, but we will omit such dependence. The support of $F_{\mathbf{b}_{-i}}(\beta)$ will be denoted $S_{\mathbf{b}_{-i}}$. Obviously, $S_{\mathbf{b}_{-i}} \subseteq\left[b_{*}, b^{*}\right]$.

Let $I \subset N$ be the set of smooth strictly increasing functions $\mathbf{b}_{i}:[0,1] \rightarrow \mathbb{R}$. Observe that if $\mathbf{b}_{-i} \in I^{n-1}$, then the distribution $F_{\mathbf{b}_{-i}}(\beta)$ is absolutely continuous with respect to the Lebesgue measure (in particular, there is no relevant tie with positive probability).

If the allocation rule is fixed and so is the profile $\mathbf{b}_{-i}$, the (interim) expected payoff of bidder $i$ of type $t_{i}$, when bidding $b_{i} \in[\underline{b}, \bar{b}]$ is:

$$
\begin{aligned}
\Pi_{i}\left(t_{i}, b_{i}, \mathbf{b}_{-i}\right) & \equiv \int\left[\bar{u}_{i}\left(t, b_{i}, \mathbf{b}_{-i}\left(t_{-i}\right)\right) a_{i}\right. \\
& \left.+\underline{u}_{i}\left(t, b_{i}, \mathbf{b}_{-i}\left(t_{-i}\right)\right)\left(1-a_{i}\right)\right] d t_{-i}
\end{aligned}
$$

\footnotetext{
${ }^{11}$ One way to see compactness is to remember Helly's Theorem, which says that a sequence of non-decreasing functions has a subsequence that converges pointwise to a nondecreasing function for all the continuity points of the limit function. The pointwise convergence implies the convergence in $\mathcal{L}^{1}$. Thus, the representative function in each equivalence class $\mathbf{b}_{i}^{m} \in N$ has a convergent subsequence that converges to $\mathbf{b}_{i} \in N$. Another way to see this is to prove that $N$ is totally bounded, constructing, for each $\varepsilon>0$, a finite covering of $N$ with sets of diameter less than $\varepsilon$. This can be done with step functions for a sufficiently fine grid.
} 
where we omitted the dependence on $a_{i}$ to simplify notation. Assume that $\Pi_{i}\left(t_{i}, b_{i}, \mathbf{b}_{-i}\right)=$ 0 if $b_{i} \in\left\{b_{O U T}^{B}, b_{O U T}^{S}\right\}$.

When $\mathbf{b}_{-i}$ is clear, we will write $\Pi_{i}\left(t_{i}, b_{i}\right)$ for $\Pi_{i}\left(t_{i}, b_{i}, \mathbf{b}_{-i}\right)$ and omit the arguments and the measure $\left(d t_{-i}\right)$.

Observe also that if $\mathbf{b}_{-i}$ do not involve ties with positive probability, then all fair allocation rules produce the same payoff. Thus, in this case, the function $\Pi_{i}\left(t_{i}, b_{i}, \mathbf{b}_{-i}\right)$ is well defined for a fair auction with unspecified tie-breaking rule (see definition 3).

Let $u_{i} \equiv \bar{u}_{i}-\underline{u}_{i}$ be the net ex-post payoff and let

$$
V_{i}\left(\mathbf{b}_{i}, \mathbf{b}_{-i}\right)=\int \Pi_{i}\left(t_{i}, \mathbf{b}_{i}\left(t_{i}\right), \mathbf{b}_{-i}\right) d t_{i}
$$

be the ex-ante payoff.

Finally, we define the interim and the ex-ante best-reply correspondence, respectively, by

and

$$
\Theta_{i}\left(t_{i}, \mathbf{b}_{-i}\right) \equiv \arg \max _{\beta \in \mathcal{B}} \Pi_{i}\left(t_{i}, \beta, \mathbf{b}_{-i}\right),
$$

$$
\Gamma_{i}\left(\mathbf{b}_{-i}\right) \equiv \arg \max _{\mathbf{b}_{i} \in \mathcal{L}^{1}([0,1], \mathcal{B})} V_{i}\left(\mathbf{b}_{i}, \mathbf{b}_{-i}\right) .
$$

\section{Existence of Pure Strategy Equilibrium}

Our first result is related to Proposition 1 of Maskin and Riley (2000). This proposition says that if there is a best reply, it is monotonic, but the authors proved it for first price auctions only. Theorem 1 says that there exists a monotonic best reply to strategies which does not involve relevant ties or when the allocation rule is smooth. Moreover, it says that all best replies are monotonic, with the only possible exception being bids out of the support of the threshold bid $T B(\cdot)$ (see Remark 1 below).

Theorem 1. Fix an auction $\mathcal{A}^{a}=\left(P,\left\{\bar{u}_{i}, \underline{u}_{i}\right\}_{i \in P}, n_{o}, \mathcal{B}, \mathcal{S}\right.$, a) satisfying Assumptions 1-4, where $a$ is a fair allocation rule or a smooth allocation rule. Fix a profile $\mathbf{b}_{-i}$ such that the distribution $F_{\mathbf{b}_{-i}}(\beta)$ is absolutely continuous with respect to the Lebesgue measure (in particular, there is no relevant tie with positive probability). Then, for each $t_{i}, \Theta_{i}\left(t_{i}, \mathbf{b}_{-i}\right)$ is non-empty. Moreover, let $t_{i}^{1}<t_{i}^{2}$ and let $b_{i}^{k}$ be a best-reply for type $t_{i}^{k}$, for $k=1,2$, that is, $b_{i}^{1} \in \Theta_{i}\left(t_{i}^{1}, \mathbf{b}_{-i}\right)$ and $b_{i}^{2} \in \Theta_{i}\left(t_{i}^{2}, \mathbf{b}_{-i}\right)$. If there are relevant bids of opponents between $b_{i}^{1}$ and $b_{i}^{2}$, that is,

$$
\operatorname{Pr}\left(\left\{t_{-i}: a_{i}\left(b_{i}^{2}, \mathbf{b}_{-i}\left(t_{-i}\right)\right) \neq a_{i}\left(b_{i}^{1}, \mathbf{b}_{-i}\left(t_{-i}\right)\right)\right\}\right)>0,
$$

then $b_{i}^{1} \leq b_{i}^{2}$.

The proof of Theorem 1 is given in the appendix. In the case of smooth allocation rules, the proof of Theorem 1 is easy to understand. In this case, the interim payoff is just the integral of its derivative on $b_{i}::^{12}$

$$
\partial_{b_{i}}\left[u_{i}(t, b) a_{i}(b)+\underline{u}_{i}(t, b)\right]=\partial_{b_{i}} u_{i}(t, b) a_{i}(b)+u_{i}(t, b) \partial_{b_{i}} a_{i}(b)+\partial_{b_{i}} \underline{u}_{i}(t, b) .
$$

\footnotetext{
${ }^{12}$ Remember that $u_{i} \equiv \bar{u}_{i}-\underline{u}_{i}$.
} 
It is easy to see that the assumptions imply that this is is strictly increasing in $t_{i}$. Thus, under the maintained assumptions, the difference $\Pi_{i}\left(t_{i}, b_{i}^{1}\right)-\Pi_{i}\left(t_{i}, b_{i}^{2}\right)$ is also increasing in $t_{i}$, because it is the integral of the above derivative over a set of positive probability. Since $b_{i}^{1}$ is optimum for a player with type $t_{i}^{1}, \Pi_{i}\left(t_{i}^{1}, b_{i}^{1}\right) \geqslant$ $\Pi_{i}\left(t_{i}^{1}, b_{i}^{2}\right)$. These two facts imply that $\Pi_{i}\left(t_{i}^{2}, b_{i}^{1}\right)>\Pi_{i}\left(t_{i}^{2}, b_{i}^{2}\right)$, but this contradicts the optimality of $b_{i}^{2}$ for a player with type $t_{i}^{2}$. In the case of fair allocation rules, the proof is just an adaptation of these ideas.

When $\Pi_{i}\left(t_{i}, b_{i}^{1}\right)-\Pi_{i}\left(t_{i}, b_{i}^{2}\right)$ is (strictly) increasing in $t_{i}$, Topkis (1978) and Vives (1990) say that $\Pi_{i}$ has (strictly) increasing differences. This property was weakened by Milgrom and Shannon (1994) to the requirement that $\Pi_{i}\left(t_{i}, b_{i}^{1}\right)-\Pi_{i}\left(t_{i}, b_{i}^{2}\right)$, as a function of $t_{i}$, crosses zero only once and from below. They called it the single crossing condition. Athey (2001) used the Milgrom-Shannon single crossing property to prove the existence of equilibrium in games of incomplete information.

Remark 1. Theorem 1 has an important consequence: the best-reply map $\Gamma_{i}\left(\mathbf{b}_{-i}\right)$ is a "unitary" set in $N$, in the following sense. Under the maintained assumptions, Theorem 1 implies that the sets $\Theta_{i}\left(t_{i}, \mathbf{b}_{-i}\right)$ and $\Theta_{i}\left(t_{i}^{\prime}, \mathbf{b}_{-i}\right)$ have at most one point in common if $t_{i} \neq t_{i}^{\prime}$. Thus, the set of types $t_{i}$ where $\Theta_{i}\left(t_{i}, \mathbf{b}_{-i}\right)$ has a diameter greater than $\varepsilon>0$ is finite. Then, $\Theta_{i}\left(t_{i}, \mathbf{b}_{-i}\right)$ is uni-valued except for a countable set of types $t_{i}$. Thus, the correspondence $t_{i} \longmapsto \Theta_{i}\left(t_{i}, \mathbf{b}_{-i}\right)$ has a unique selection in $\mathcal{L}^{1}$. By the definition of $\Gamma_{i}\left(\mathbf{b}_{-i}\right)$, we conclude that this selection is the unique function in $\Gamma_{i}\left(\mathbf{b}_{-i}\right)$, and it is non-decreasing. Nevertheless, this is true only when

$$
\operatorname{Pr}\left(\left\{t_{-i}: a_{i}\left(\mathbf{b}_{i}^{2}\left(t_{i}\right), \mathbf{b}_{-i}\left(t_{-i}\right)\right) \neq a_{i}\left(\mathbf{b}_{i}^{1}\left(t_{i}\right), \mathbf{b}_{-i}\left(t_{-i}\right)\right)\right\}\right)>0,
$$

for two functions $\mathbf{b}_{i}^{2}$ and $\mathbf{b}_{i}^{2}$ in the set of best replies. If the two functions induce winning and losing in the same events (for instance, functions that specify nonserious bids, that is, bids below those of the opponents), then it is not always true that the convex combination of best replies is also a best reply (but this is true under Assumption 5, as we show in the proof of Lemma 3).

This remark clarifies that Theorem 1 cannot be obtained from Topkis (1978)'s Theorem 6.3 or Milgrom and Shannon (1994)'s Theorem 4', which ensure that all selections of the best-reply map are non-decreasing. The reason is that such a conclusion is not true in general. Also, it points out that strict single crossing or strict increasing differences are not necessarily true in our setting.

Theorem 1 can be used to obtain the following:

Theorem 2. Fix a fair auction with unspecified tie-breaking rule $\mathcal{A}=\left(P,\left\{\bar{u}_{i}, \underline{u}_{i}\right\}_{i \in P}\right.$, $\left.n_{o}, \mathcal{B}\right)$ satisfying Assumptions 1-4. Then there exists a specification $\mathcal{A}^{*}=(P$, $\left.\left\{\bar{u}_{i}, \underline{u}_{i}\right\}_{i \in P}, n_{o}, \mathcal{B}, \mathcal{S}, a^{*}\right)$, where $a^{*}: \mathcal{B}^{n} \times[0,1]^{n} \rightarrow\{0,1\}^{n}$ is a fair allocation rule which requires the announcement of types to break ties, such that there exists a pure strategy equilibrium $\left(\mathbf{b}^{*}, \mathbf{s}^{*}\right)$ for $\mathcal{A}^{*}$, where $\mathbf{b}^{*}$ is a profile of non-decreasing functions and $\mathbf{s}^{*}$ is the identity (that is, the bidders announce their types truthfully). Moreover, for each $i=1, \ldots, n, a_{i}^{*}\left(\mathbf{b}^{*}(t), \mathbf{s}^{*}(t)\right)$ is non-decreasing in $t_{i}$ and non-increasing in $t_{-i}$.

The proof of Theorem 2 is given in subsection 3.2 and its idea is as follows. First, we introduce a sequence of smooth allocation rules that approximate fair allocation 
rules. Using Theorem 1, it is easy to obtain the existence of equilibrium for each auction defined with a smooth allocation rule in this sequence. Next, we consider the sequence of these equilibria. Since the equilibria are in the compact set $N$, they have a convergent subsequence. Using such a subsequence, we define a tie-breaking rule that sustains the limit of equilibria as the equilibrium of the original auction. The proof is concluded by arguing that the definition of the tie-breaking rule ensures that a profitable deviation at the limit would imply a profitable deviation throughout the sequence of equilibria, which is absurd.

One question that can arise is whether we could use Athey's proof to obtain our results. Theorem 2 cannot be derived from Athey (2001)'s Theorems 6 or 7, for two reasons. First, her model does not allow the dependence of the utility on the bid of opponents as ours does. Second, her assumption A3 is not satisfied by the models that we consider (see discussion in subsection 3.1 below).

An important point about Theorem 2 is why we need a special tie-breaking rule (that is, a rule distinct of the standard tie-breaking rule), which is not so usual in auction theory. In the following subsection, we discuss why the standard tie-breaking rule is not sufficient for equilibrium existence.

3.1. Why a special tie-breaking rule? Since we are treating single and double auctions, the fact that the standard tie-breaking rule (breaking the tie randomly) is not sufficient to ensure equilibrium existence is easy to establish. Indeed, consider the following example:

Example 1. (Jackson and Swinkels, 2005, Example 4) There are two players, a buyer and a seller, and the payment is the medium of the two bids. The value of the object for the seller is uniformly distributed on $[0,1]$, that $i s, \bar{u}_{1}(t, b)=t_{1}$, and she receives the price if she sells: $\underline{u}_{1}(t, b)=\left(b_{1}+b_{2}\right) / 2$. The value of the object for the buyer is uniformly distributed on $[3,4]$, that is, $\bar{u}_{2}(t, b)=3+t_{2}-\left(b_{1}+b_{2}\right) / 2$ and the buyer pays nothing if she loses, $\underline{u}_{2}(t, b)=0$. Jackson and Swinkels (2005) prove that there is no equilibrium for this auction under the standard tie-breaking rule.

Although there is no equilibrium for the example above under the standard tiebreaking rule, it exists under a trade-maximixing tie-breaking rule, that is, a rule that specifies that a tie between a buyer and a seller is solved by giving the object to the buyer. Although Jackson and Swinkels (2005) show that this is sufficient for private value auctions, the same is not necessarily true for interdependent value double auctions. Moreover, even for single-sided auctions, the generality of our assumptions implies that the equilibrium existence may require special tie-breaking rules. To see this, consider the following example:

Example 2. (JSSZ, Example 1) There are two buyers disputing one object. For $i=1,2$, consider $\bar{u}_{i}(b, t)=5+t_{i}-4 t_{-i}-b_{i}$ and $\underline{u}_{i}(b, t)=0$. Observe that this example satisfies all Assumptions 1-5. JSSZ prove that this auction does not have an equilibrium under the standard tie-breaking rule. (See also Jackson, Swinkels, Simon and Zame, 2004).

Since this example satisfies our assumptions, it is not possible to provide an existence result that does not need a special tie-breaking rule. On the other hand, 
Athey (2001) proves the existence of equilibrium under the standard tie-breaking for a class of discontinuous games. Thus, it is useful to understand why her Theorem 6 does not apply to the above example. Indeed, this example satisfies all of her assumptions but Assumption A3, which requires that the expected value of $u_{i}\left(t_{i}, b_{i}, \cdot\right)$ conditioned on the event of winning with a bid $\beta$ is nondecreasing in $\beta$ (maintaining $b_{i}$ fixed), when the opponents follow non-decreasing strategies. It is easy to see why Example 2 does not satisfy such an assumption: if the opponents are following nondecreasing strategies, increasing $\beta$, bidder $i$ will win against opponents of a higher type, which implies that her utility may decrease.

It is also easy to see that Athey's assumption A3 is not valid for double auctions: increasing $\beta$ may imply that a buyer wins against sellers who are asking higher prices, which may imply a higher price to be paid and, consequently, a decrease of the expected utility. This explains why we need special tie-breaking rules for the auctions which we consider.

It is an open question whether it is possible to ensure the equilibrium existence result in our setting with a tie-breaking that does not require announcement of types. Nevertheless, in the symmetrical case, Araujo, de Castro and Moreira (2006) show that an all-pay auction tie-breaking rule is sufficient to solve the problem of equilibrium existence. This rule does not require the annoucement of types and is inspired by the Vickrey auction tie-breaking rule used by Maskin and Riley (2000).

Although we do not have a complete characterization of the tie-breaking rules, Theorem 2 provides a partial characterization: the tie-breaking rule is monotonic in the types.

3.2. Proof of Theorem 2. The proof of Theorem 2 is given through a series of lemmas, whose proofs are in the appendix. We begin by the following fact, whose proof follows the idea of the existence of partition of unity:

Lemma 2. For each $m \in\{2,3, \ldots\}$, there exists a $C^{\infty}$ allocation rule $a^{m}: \mathcal{B}^{n} \rightarrow$ $[0,1]^{n}$ (set $\mathcal{S}=\{\varnothing\}$ in the original definition), such that: (i) if $b_{i}>T B\left(b_{-i}\right)+\frac{1}{m}$, then $a_{i}^{m}(b)=1$ and; (ii) if if $b_{i}<T B\left(b_{-i}\right)-\frac{1}{m}$, then $a_{i}^{m}(b)=0 .{ }^{13}$

For each $m \in\{2,3, \ldots\}$, consider the auction game $\mathcal{A}^{m}=\left(P,\left\{\bar{u}_{i}, \underline{u}_{i}\right\}_{i \in P}, n_{o}, \mathcal{B}\right.$, $\mathcal{S}=\{\varnothing\}$, obtained from $\mathcal{A}$ by specifying the smooth allocation rules $a^{m}$ given by the previous lemma.

Lemma 3. For each $m, \mathcal{A}^{m}$ has an equilibrium in non-decreasing strategies. ${ }^{14}$

Let us denote by $\mathbf{b}^{m}=\left(\mathbf{b}_{1}^{m}, \ldots, \mathbf{b}_{n}^{m}\right)$ the equilibrium of the auction $\mathcal{A}^{m}$. Since $\left\{\mathbf{b}^{m}\right\} \subset N^{n}$, it has a convergent subsequence. Passing to it (without renaming), we have: $\mathbf{b}^{m} \rightarrow \mathbf{b}^{*}$. We show below that $\mathbf{b}^{*}$ is equilibrium of the auction $\mathcal{A}^{*}=(P$, $\left.\left\{\bar{u}_{i}, \underline{u}_{i}\right\}_{i \in P}, n_{o}, \mathcal{B}, \mathcal{S}=[0,1], a^{*}\right)$, where $a^{*}: \mathcal{B}^{n} \times \mathcal{S}^{n} \rightarrow[0,1]$ is a fair allocation rule that depends, for the case of relevant ties, on the announcement of types $s \in$

\footnotetext{
${ }^{13}$ Note that these allocation rules are not fair, in the sense of definition 2.

${ }^{14}$ We use Assumption 5 only in the proof of this lemma. Nevertheless, the lemma is valid without it, by Theorem 6.1 of Vives (1990) or by Corollary 2.1 of Athey (2001).
} 
$\mathcal{S}^{n}=[0,1]^{n}$. The allocation rule $a^{*}$ is defined as follows, if there is a relevant tie for bidder $i$ (that is, $\left.b_{i}=T B\left(b_{-i}\right)\right)$ and $s=\left(s_{i}, s_{-i}\right)$ are the announced types:

$$
a_{i}^{*}(b, s)= \begin{cases}\lim _{m} a_{i}^{m}\left(\mathbf{b}^{m}(s)\right), & \text { if } b_{j}=\lim \mathbf{b}_{j}^{m}\left(s_{j}\right), \text { for all } j \\ \lim _{m} a_{i}^{m}(b), & \text { otherwise }\end{cases}
$$

The next result concludes the demonstration of Theorem 2 .

Lemma 4. Consider the auction $\mathcal{A}^{*}=\left(P,\left\{\bar{u}_{i}, \underline{u}_{i}\right\}_{i \in P}, n_{o}, \mathcal{B}, \mathcal{S}=[0,1], a^{*}\right)$ and $\mathbf{b}^{*}$ as defined above. Let $\mathbf{s}^{*}:[0,1]^{n} \rightarrow[0,1]^{n}$ be the identity, that is, $\mathbf{s}_{i}^{*}\left(t_{i}\right)=t_{i}$ is the strategy of announcing the own type truthfully. If the opponents are following $\left(\mathbf{b}_{-i}^{*}, \mathbf{s}_{-i}^{*}\right)$, it is optimum for bidder $i$ to follow $\left(\mathbf{b}_{i}^{*}, \mathbf{s}_{i}^{*}\right)$.

\section{Positive probability of trade}

In this section we prove that there is a non-trivial equilibria for double auctions. Besides the specification contained in Assumption 6, we will need the following:

Assumption 7. The sets of sellers $S=\left\{1,2, \ldots, n_{S}\right\}$ and buyers $B=\left\{n_{S}+1, \ldots, n\right\}$ are non-empty and they have the utilities specified in Assumption 6. Moreover, for all $b \in \mathcal{B}^{n}$, there exists $K, L^{\prime}$ and $L$ such that:

(i) for all $i \in P, 0<L^{\prime} \leq U_{i}^{\prime} \leq L$;

(ii) for all $i \in P, 0 \leq \partial_{b_{i}} p_{i}\left(b_{i}, b_{-i}\right) \leq K$;

(iii) $p_{i}(b) \in\left[\max \left\{\underline{b}, \min _{j=1, \ldots, n} b_{j}\right\}, b_{i}\right]$ for all $i \in B$;

(iv) $p_{j}(b) \in\left[b_{j}, \min \left\{\bar{b}, \max _{j=1, \ldots, n} b_{j}\right\}\right]$ for all $j \in S$;

(v) if $b_{i}=T B\left(b_{-i}\right)$, then $p_{i}(b)=b_{i}$, for all $i \in P$.

Items (i) and (ii) of Assumption 7 are limitations of derivatives of utility and payment functions, $U_{i}$ and $p_{i}$, respectively; item (iii) says a buyer pays at most her bid; item (iv) says that a seller receives at least her bid; and item (v) specifies that the payment is the value of the bid in case of a relevant tie. These are natural assumptions to require.

For each $i \in P$, let $\bar{w}_{i}\left(t_{i}\right)$ be the maximum of possible values of the object for player $i$ with type $t_{i}$, that is, $\bar{w}_{i}\left(t_{i}\right) \equiv \sup \left\{v_{i}\left(t_{i}, t_{-i}\right): t_{-i} \in[0,1]^{n-1}\right\}$. Similarly, let $\underline{w}_{i}\left(t_{i}\right)$ be the minimum of possible values of the object for player $i$ with type $t_{i}$, that is, $\underline{w}_{i}\left(t_{i}\right) \equiv \inf \left\{v_{i}\left(t_{i}, t_{-i}\right): t_{-i} \in[0,1]^{n-1}\right\}$. It is clear that $\bar{w}_{i}$ and $\underline{w}_{i}$ are non-decreasing functions. Let $\bar{w}$ be the maximum possible value for buyers and let $\underline{w}$ be the minimum possible value for sellers, that is, $\bar{w} \equiv \max _{i \in B} \bar{w}_{i}(1)$ and $\underline{w} \equiv \min _{j \in S} \underline{w}_{j}(0)$.

We need the following:

Assumption 8. (i) $\underline{b} \leq \underline{w}<\bar{w} \leq \bar{b}$ and (ii) there exist a seller $j \in S$, a buyer $i \in B$ and types $t_{i}, t_{j} \in(0,1)$ such that $\underline{w}_{i}\left(t_{i}\right)>\underline{w}$ and $\bar{w}_{j}\left(t_{j}\right)<\bar{w}$.

The first part of this assumption requires that the maximum value for buyers be above the minimum value for sellers. This part of the assumption is weak and natural. Indeed, if we have $\underline{w} \geq \bar{w}$, trade will be profitable for the players with probability zero (from the atomless assumption on the values). 
The second part of the assumption is not trivial for interdependent value auctions, although it is a consequence of the first part for private value auctions (where $\left.\underline{w}_{i}\left(t_{i}\right)=\bar{w}_{i}\left(t_{i}\right)\right)$. It requires that there exists a type of a buyer (it can be a very high type, close to 1) whose minimum value is above the minimum value for sellers; and a type of a seller (it can be a type very close to 0) whose maximum value is below the maximum value for buyers. For interdependent value auctions, only condition (i) of Assumption 8 is not sufficient to ensure the existence of trade, as the next example shows.

Example 3. There is one seller (player 1) and one buyer (player 2); the players are risk-neutral (that is, $U_{1}(x)=U_{2}(x)=x$ ), the value of the object for the seller is uniformly distributed on [2,3] and privately known by the seller (that is, $v_{1}\left(t_{1}, t_{2}\right)=$ $\left.t_{1}+2\right)$ and the value for the buyer is given by $v_{2}\left(t_{1}, t_{2}\right)=3 t_{1}+t_{2}$. It is a first price auction for both players (the auctioneer pockets the difference of the bids). It is easy to see that $\underline{w}=2<\bar{w}=4$. Thus, the first part of Assumption 8 is satisfied. Also, $\bar{w}_{1}\left(t_{1}\right)=t_{1}+2 \leq 3<4=\bar{w}$, which is exactly the condition $\bar{w}_{j}\left(t_{j}\right)<\bar{w}$ in item (ii) of Assumption 8. Nevertheless, $\underline{w}_{2}\left(t_{2}\right)=0<\underline{w}=1$ for all $t_{1} \in[0,1]$, so that the second part of Assumption 8 is not satisfied. This auction has no equilibrium (even in mixed strategies) with positive probability of trade. To see this, assume that there is a mixed strategy equilibrium with positive probability of trade. Let $\beta$ be a bid for which there is positive probability of negotiation. The seller will accept such an offer only if $v_{1}\left(t_{1}, t_{2}\right)=t_{1}+2<\beta$ (the event $v_{1}\left(t_{1}, t_{2}\right)=\beta$ has zero measure and the behavior in it does not matter). Thus, the object for the buyers that negotiate under $\beta$ has expected value not greater than:

$E\left[\left(3 t_{1}+t_{2}-\beta\right) 1_{\left[t_{1}+2 \leq \beta\right]}\right]= \begin{cases}0, & \text { if }(\beta-2)<0 \\ \frac{3}{2}(\beta-2)^{2}+\left(t_{2}-\beta\right)(\beta-2), & \text { if }(\beta-2) \in[0,1] \\ t_{2}+\frac{3}{2}-\beta & \text { if }(\beta-2)>1\end{cases}$

Observe that the last line is negative for $\beta>3$ (that is, $\beta-2>1$ ), while the maximum value of the second line for $\beta \in[2,3]$ is 0 , achieved by $\beta=2$ (and the expected value is negative for all $\beta \in(2,3])$. Thus, it is better for the buyer to bid $\beta \leq 2$, which wins with zero probability.

The reader should note that the above example has some of the characteristics of Akerlof's (1970) famous "market for lemons" example. The seller is better informed than the buyer about the value of the object to the buyer; the buyer does not know the value for the seller, and the range of possible prices excludes the more valuable sellers.

The logic behind the example explains why we need Assumption 8: simply, the value of the object for the buyers is such that they cannot guarantee profits with any bid because their values are too dependent on the values of the sellers. Assumption 8 requires them to ensure a value at least above the least value of the seller. In such a way, we manage to guarantee that there exists a price that is good for both parties. The example also shows that it is not sufficient to assume only one of the two conditions: either $\underline{w}_{i}\left(t_{i}\right)>\underline{w}$ or $\bar{w}_{j}\left(t_{j}\right)<\bar{w}$. 
We have the following:

Theorem 3. Fix an auction $\mathcal{A}$ satisfying Assumptions 1 and 6-8. Then, there is an equilibrium where trade occurs with positive probability.

4.1. Proof of Theorem 3. Our proof will follow the same argument as the proof of Theorem 15 of Jackson and Swinkels (2005). For $x \in\{3,4, \ldots\}$, consider an auction $\mathcal{A}^{x}$ modified from $\mathcal{A}$ as follows: besides the strategic players $1,2, \ldots, n$, there are two non-strategic players, 0 and $n+1$, a seller and a buyer, who play a bid uniformly distributed on $[\underline{w}, \bar{w}]$ with probability $\frac{1}{x}$, and stay out of the auction with probability $1-\frac{1}{x} \cdot 15$

Our existence result ensures the equilibrium existence for auction $\mathcal{A}^{x}$ (remember that Lemma 1 says that Assumption 6 implies Assumptions 2-5). Let $\mathbf{b}^{x}$ be a pure strategy monotonic equilibrium of $\mathcal{A}^{x}$. Now consider, as before, the limit $\mathbf{b}^{x} \rightarrow \mathbf{b}^{*}$ as $x \rightarrow \infty$. The proof of Theorem 2 can be used to argue that $\mathbf{b}^{*}$ is again an equilibrium of the original auction with an appropriate fair allocation rule.

Clearly, the probability of trade is continuous in $\mathbf{b}$ and is positive for each auction $\mathcal{A}^{x}$. The argument consists in finding a contradiction if the probability of trade goes to zero when $x \rightarrow \infty$. We first prove that if the probability of trade goes to zero, then the probability of bids by buyers above $\bar{w}-2 \delta$ goes to zero, as does the probability of bids by sellers below $\underline{w}+2 \delta$. The contrapositive of this is the content of the following lemma.

Lemma 5. Fix $0<2 \delta<\min \left\{\underline{w}_{i}\left(t_{i}\right)-\underline{w}, \bar{w}-\bar{w}_{j}\left(t_{j}\right)\right\}$, for $i \in B, j \in S$ and types $t_{i}, t_{j} \in(0,1)$ given by the Assumption 8. Assume that there is a subsequence of auctions $\mathcal{A}^{x}$ such that equilibrium profiles $\mathbf{b}^{x}$ specify a probability $\gamma>0$ of bids by sellers below $\underline{w}+2 \delta$ (alternatively, of bids by buyers above $\bar{w}-2 \delta$ ). Then, the probability of trade along this subsequence is bounded away from zero.

The fact that these probabilities go to zero is now used to argue that there is a player $j$ with a profitable deviation $\mathbf{d}_{j}$ in auction $\mathcal{A}^{x}$, which contradicts $\mathbf{b}^{x}$ being equilibrium. To reach the contradiction, we need a characterization of profitable deviations in our setting, which the following lemmas provide.

Lemma 6. Fix a buyer $i \in B$, a type $t_{i} \in[0,1]$ and a profile $\mathbf{b}_{-i}$ of strategies followed by buyer $i$ 's opponents, that is, players $j \neq i$. Consider the deviation from a bid $\beta_{1}$ to the bid $\beta_{2}>\beta_{1}$. The deviation is advantageous to buyer $i$ if

$$
L^{\prime}\left(\underline{w}_{i}\left(t_{i}\right)-\beta_{2}\right) \operatorname{Pr}\left[a_{i}\left(\beta_{2}, \cdot\right)>a_{i}\left(\beta_{1}, \cdot\right)\right]>L K\left(\beta_{2}-\beta_{1}\right) \operatorname{Pr}\left[a_{i}\left(\beta_{1}, \cdot\right)>0\right] .
$$

Lemma 7. Fix a seller $j \in S$, a type $t_{j} \in[0,1]$ and a profile $\mathbf{b}_{-j}$ of strategies followed by seller $j$ 's opponents. Consider the deviation from a bid $\beta_{1}$ to the bid $\beta_{2}<\beta_{1}$. The deviation is advantageous to seller $j$ if

$$
L^{\prime}\left(\beta_{2}-\bar{w}_{j}\left(t_{j}\right)\right) \operatorname{Pr}\left[a_{i}\left(\beta_{1}, \cdot\right)>a_{i}\left(\beta_{2}, \cdot\right)\right]>L K\left(\beta_{1}-\beta_{2}\right) \operatorname{Pr}\left[a_{i}\left(\beta_{1}, \cdot\right)<1\right] .
$$

\footnotetext{
${ }^{15}$ The strategies can be defined as follows: $\mathbf{b}_{0}^{x}\left(t_{0}\right)=x t_{0}(\bar{w}-\underline{w})+\underline{w}$, if $t_{0}<\frac{1}{x}$ and $\mathbf{b}_{0}^{x}\left(t_{0}\right)=$ $b_{O U T}^{S}$ if $t_{0} \in\left[\frac{1}{x}, 1\right] ; \mathbf{b}_{n+1}^{x}\left(t_{n+1}\right)=b_{O U T}^{B}$ if $t_{0}<1-\frac{1}{x}$ and $\mathbf{b}_{n+1}^{x}\left(t_{n+1}\right)=x\left(t_{n+1}-1+\frac{1}{x}\right)(\bar{w}-\underline{w})$ $+\underline{w}$ if $t_{0} \in\left[1-\frac{1}{x}, 1\right]$.
} 
Lemmas 5, 6 and 7 provide the results necessary to adapt the proof of Jackson and Swinkels (2005) to the setting of auctions with interdependent values. In other words, the proof of the following lemma follows closely the proof of their Theorem 15.

Lemma 8. There exists an $x$, a player $j \in P$ and a deviation $\mathbf{d}_{j}$ from $\mathbf{b}_{j}^{x}$, which is profitable for player $j$ in the auction $\mathcal{A}^{x}$.

This concludes the proof of Theorem 3.

\section{Conclusion: Related literature and the COntribution}

The main contributions of this paper are: (1) a proof of existence of monotonic pure strategy equilibrium in a setting where only the existence of a mixed strategy equilibrium is known; (2) a proof of existence of trade with positive probability for auctions with asymmetrical interdependent values.

A theorem that includes our setting is that of JSSZ, who prove the existence of asymmetrical mixed strategy equilibrium with any distribution of types. They used the "endogenously defined" tie-breaking rule solution concept introduced by Simon and Zame (1990), as we do too. We particularize their assumptions to the independent types' case, but we are able to obtain the existence in monotonic pure strategies.

Athey (2001), for general games, and Reny and Zamir (2004), for first-price auctions, obtained monotonic pure strategy equilibrium without special tie-breaking rules. Nevertheless, they assumed the monotonicity of the utilities with respect to all types and do not consider double auctions, as we do.

Williams (1991) consider symmetric double auctions with independent types. Jackson and Swinkels (2005) consider (multi-unit) asymmetrical double auctions with general distribution of types, but they are restricted to the private value case. In this setting, they are able to prove that the tie-breaking rule does not matter, a result that does not hold in our setting, as we argued in subsection 3.1. Reny and Perry (2003) and Fundenberg, Mobius and Szeil (2003) consider symmetrical double auctions with conditionally independent types but are only able to prove the existence of equilibrium when the number of players is high. Thus, these works do not cover our equilibrium existence result for asymmetrical double auctions with interdependent values and small number of players.

Also, the previous results ensuring the existence of pure strategy equilibrium do not consider utilities that may be decreasing in the signal of opponents. Thus, they do not need special tie-breaking rules as our setting requires.

Our method of proof is related to that used by Fundenberg, Mobius and Szeil (2003). They also used a perturbation of the allocation rule and worked with a compact space of functions. Their space is that of functions near the identity. Accordingly, they do not need special tie-breaking rules, but do need a large number of players, which is not necessary in our case.

Another point is that our proof clarifies that is not necessary at all to discretize the space of bids and this simplifies the proof. We also show that the argument of convexity can be made straightforwardly, if we require Assumption 5. 
The other main contribution of the paper is the result concerning positive probability of trade. While Jackson and Swinkels (2005) established it for a general model of private value auctions, we show by a counterexample that this result is not necessarily true for interdependent value auctions. Introducing an appropriate condition, we are able to prove the non-triviality of the equilibrium.

\section{Appendix}

The proof of Theorem 1 requires the following lemma.

Lemma 1. Assume (A0). Fix a profile of bidding functions $\mathbf{b}_{-i}$ such that $F_{\mathbf{b}_{-i}}(\beta)$ is absolutely continuous with respect to the Lebesgue measure and $a$ is a fair allocation rule. The payoff of bidder $i$ can be expressed by

$$
\Pi_{i}\left(t_{i}, b_{i}, \mathbf{b}_{-i}\right)=\Pi_{i}\left(t_{i}, b_{*}, \mathbf{b}_{-i}\right)+\int_{\left[b_{*}, b_{i}\right)} \partial_{b_{i}} \Pi_{i}\left(t_{i}, \beta, \mathbf{b}_{-i}\right) d \beta .
$$

where $\partial_{b_{i}} \Pi_{i}\left(t_{i}, \beta\right)$ exists for almost all $\beta \in\left(b_{*}, b^{*}\right)$ and is given by

$$
\begin{aligned}
\partial_{\beta} \Pi_{i}\left(t_{i}, \beta, \mathbf{b}_{-i}\right)= & E\left[\partial_{b_{i}} \bar{u}_{i}\left(t_{i}^{1}, \cdot\right) 1_{\left[\beta>T B\left(\mathbf{b}_{-i}(\cdot)\right)\right]}\right] \\
& +E\left[\partial_{b_{i}} \underline{u}_{i}\left(t_{i}^{1}, \cdot\right) 1_{\left[\beta<T B\left(\mathbf{b}_{-i}(\cdot)\right)\right]}\right] \\
& +E\left[u_{i}\left(t, \beta, \mathbf{b}_{-i}\left(t_{-i}\right)\right) \mid T B\left(\mathbf{b}_{-i}(\cdot)\right)=\beta\right] f_{\mathbf{b}_{-i}}(\beta) .
\end{aligned}
$$

This lemma can be proved using the Leibiniz rule. For a proof in a more general setting, see de Castro and Riascos (2004). Now, we proceed to the proof of Theorem 1.

Proof of Theorem 1. Let us first consider a fair allocation rule. Under the assumption on $\mathbf{b}_{-i}, \Pi_{i}\left(t_{i}, b_{i}, \mathbf{b}_{-i}\right)$ is continuous. Since $\mathcal{B}$ is compact, $\Theta_{i}\left(t_{i}, \mathbf{b}_{-i}\right)$ is obviously non-empty (and compact). For the second part, assume that $t_{i}^{1}<t_{i}^{2}$, $b_{i}^{1} \in \Theta_{i}\left(t_{i}^{1}, \mathbf{b}_{-i}\right), b_{i}^{2} \in \Theta_{i}\left(t_{i}^{2}, \mathbf{b}_{-i}\right), b_{i}^{2}<b_{i}^{1}$ and

$$
\operatorname{Pr}\left(\left\{t_{-i}: a_{i}\left(b_{i}^{2}, \mathbf{b}_{-i}\left(t_{-i}\right)\right)<a_{i}\left(b_{i}^{1}, \mathbf{b}_{-i}\left(t_{-i}\right)\right)\right\}\right)>0 .
$$

Since $[0,1]^{n-1}$ and $\mathcal{B}^{n}$ are compact and $u_{i}$ is (absolutely) continuous, there exists $\delta>0$ such that $u_{i}\left(t_{i}^{1}, t_{-i}, b\right)+2 \delta<u_{i}\left(t_{i}^{2}, t_{-i}, b\right)$ for all $t_{-i} \in[0,1]^{n-1}$ and all $b \in B^{n}$. For a bid $\beta \in B$, define the functions

$$
\begin{aligned}
& g^{1}\left(t_{-i}\right)=u_{i}\left(t_{i}^{1}, t_{-i}, \beta, \mathbf{b}_{-i}\left(t_{-i}\right)\right), \text { and } \\
& g^{2}\left(t_{-i}\right)=u_{i}\left(t_{i}^{2}, t_{-i}, \beta, \mathbf{b}_{-i}\left(t_{-i}\right)\right) .
\end{aligned}
$$

Then, $g^{1}\left(t_{-i}\right)+2 \delta<g^{2}\left(t_{-i}\right)$. By the positivity of conditional expectations, ${ }^{16}$

$$
E\left[g^{2}-g^{1}-2 \delta \mid T B\left(\mathbf{b}_{-i}(\cdot)\right)=\beta\right] \geqslant 0 .
$$

Thus, from the independence of types, we conclude that

$$
E\left[u_{i}\left(t_{i}^{1}, \cdot\right) \mid T B\left(\mathbf{b}_{-i}(\cdot)\right)=\beta\right]+\delta<E\left[u_{i}\left(t_{i}^{2}, \cdot\right) \mid T B\left(\mathbf{b}_{-i}(\cdot)\right)=\beta\right] .
$$

\footnotetext{
${ }^{16}$ See, for instance, Kallenberg (2002), Theorem 6.1, p. 104.
} 
By Assumption 4,

$$
E\left[\partial_{b_{i}} \bar{u}_{i}\left(t_{i}^{1}, \cdot\right) 1_{\left[\beta>T B\left(\mathbf{b}_{-i}(\cdot)\right)\right]}\right] \leqslant E\left[\partial_{b_{i}} \bar{u}_{i}\left(t_{i}^{2}, \cdot\right) 1_{\left[\beta>T B\left(\mathbf{b}_{-i}(\cdot)\right)\right]}\right] .
$$

and

$$
E\left[\partial_{b_{i}} \underline{u}_{i}\left(t_{i}^{1}, \cdot\right) 1_{\left[\beta<T B\left(\mathbf{b}_{-i}(\cdot)\right)\right]}\right] \leqslant E\left[\partial_{b_{i}} \underline{u}_{i}\left(t_{i}^{2}, \cdot\right) 1_{\left[\beta<T B\left(\mathbf{b}_{-i}(\cdot)\right)\right]}\right] .
$$

Then, (3), (4), (5) and the expression of $\partial_{b_{i}} \Pi_{i}\left(t_{i}, \beta, \mathbf{b}_{-i}\right)$ given by (1) imply that for almost all $\beta$,

$$
\partial_{b_{i}} \Pi_{i}\left(t_{i}^{2}, \beta, \mathbf{b}_{-i}\right)>\partial_{b_{i}} \Pi_{i}\left(t_{i}^{1}, \beta, \mathbf{b}_{-i}\right)+\delta f_{\mathbf{b}_{-i}}(\beta) .
$$

The difference $\Pi_{i}\left(t_{i}^{2}, b_{i}^{1}, \mathbf{b}_{-i}\right)-\Pi_{i}\left(t_{i}^{2}, b_{i}^{2}, \mathbf{b}_{-i}\right)$ can be written as the integral:

$$
\begin{aligned}
\int_{\left[b_{i}^{2}, b_{i}^{1}\right)} \partial_{b_{i}} \Pi_{i}\left(t_{i}^{2}, \beta, \mathbf{b}_{-i}\right) d \beta & >\int_{\left[b_{i}^{2}, b_{i}^{1}\right)} \partial_{b_{i}} \Pi_{i}\left(t_{i}^{1}, \beta, \mathbf{b}_{-i}\right) d \beta+\delta \int_{\left[b_{i}^{2}, b_{i}^{1}\right)} f_{\mathbf{b}_{-i}}(\beta) d \beta \\
& \geqslant \delta\left[F_{\mathbf{b}_{-i}}\left(b_{i}^{1}\right)-F_{\mathbf{b}_{-i}}\left(b_{i}^{2}\right)\right] \\
& \geqslant 0,
\end{aligned}
$$

where the first inequality comes from (2) and (6); the second comes from the fact that $b_{i}^{1} \in \Theta_{i}\left(t_{i}^{1}, \mathbf{b}_{-i}\right)$, that is,

$$
\int_{\left[b_{i}^{2}, b_{i}^{1}\right)} \partial_{b_{i}} \Pi_{i}\left(t_{i}^{1}, \beta, \mathbf{b}_{-i}\right) d \beta \geqslant 0 ;
$$

and the third comes from $b_{i}^{1}>b_{i}^{2}$. Now, this implies that $\Pi_{i}\left(t_{i}^{2}, b_{i}^{1}, \mathbf{b}_{-i}\right)>\Pi_{i}\left(t_{i}^{2}, b_{i}^{2}, \mathbf{b}_{-i}\right)$, which contradicts the fact that $b_{i}^{2} \in \Theta_{i}\left(t_{i}^{2}, \mathbf{b}_{-i}\right)$.

The proof for smooth allocation rules is similar. First, we observe that the ex-post utility $u_{i}(t, b) a_{i}(b)+\underline{u}_{i}(t, b)$ is continuous and has increasing differences in $\left(t_{i}, b_{i}\right)$, because

$$
\partial_{b_{i}} u_{i}(t, b) a_{i}(b)+u_{i}(t, b) \partial_{b_{i}} a_{i}(b)+\partial_{b_{i}} \underline{u}_{i}(t, b)
$$

is increasing in $t_{i}$. Since the expression of $\partial_{b_{i}} \Pi_{i}\left(t_{i}^{1}, \beta, \mathbf{b}_{-i}\right)$ is the integral of the above expression, we can repeat the arguments and use (2) to obtain the same conclusion.

Proof of Lemma 2. Let $a: \mathcal{B}^{n} \rightarrow[0,1]^{n}$ be any fair allocation rule and consider $n$ independent and identically distributed variables $\varepsilon_{i}$, with support contained in $\left(\frac{-1}{2 m}, \frac{1}{2 m}\right)$ and whose density function is $C^{\infty}$. Define, for each $i$,

$$
\begin{aligned}
a_{i}^{m}(b) & =E_{\left(\varepsilon_{i}\right)_{i \in P}}\left[a_{i}\left(\left(b_{i}+\varepsilon_{i}\right)_{i \in P}\right)\right] \\
& =\operatorname{Pr}\left\{\left(\varepsilon_{i}\right)_{i \in P}: b_{i}+\varepsilon_{i}>T B\left(\left(b_{j}+\varepsilon_{j}\right)_{j \neq i}\right)\right\} .
\end{aligned}
$$

Ties in the bids $\left(b_{i}+\varepsilon_{i}\right)_{i \in P}$ occur with zero probability so that the expectation above is equal across all the fair allocation rules and is well-defined.

Now, we verify conditions (i)-(iv) of Definition 1 . It is easy to see that $t \mapsto$ $a^{m}(\mathbf{b}(t))$ if $t \mapsto \mathbf{b}(t)$ is measurable. Thus, (i) is satisfied. For each realization of $\left(\varepsilon_{i}\right)_{i \in P}$, we have $\sum_{i \in P} a_{i}\left(\left(b_{i}+\varepsilon_{i}\right)_{i \in P}\right)=n_{o}$ with probability one. Thus, $\sum_{i \in P} a_{i}^{m}(b)=n_{o}$, which implies condition (ii). 
It is easy to see that $a_{i}^{m}$ is non-decreasing in $b_{i}$ and non-increasing in $b_{j}$, for $j \neq i$, which is condition (iii). Condition (iv) is trivial, since $\mathcal{S}=\{\varnothing\}$ for $a^{m}$. The Lemma is proved.

Proof of Lemma 3. Fix $m$ and define the set-valued map $\Upsilon_{i}^{m}\left(\mathbf{b}_{-i}\right) \equiv \Gamma_{i}^{m}\left(\mathbf{b}_{-i}\right) \cap$ $N$, where $\Gamma_{i}^{m}$ is the best-reply map for auction $\mathcal{A}^{m}$. For simplicity, we omit the upperscript $m$.

It is easy to see, from continuity and Theorem 1 , that $\Upsilon_{i}: N^{n-1} \rightarrow N$ is upper semicontinuous with non-empty values. Let us show that it has convex values.

Suppose that $\mathbf{b}_{i}, \mathbf{b}_{i}^{\prime} \in \Upsilon_{i}\left(\mathbf{b}_{-i}\right)$, with $\mathbf{b}_{i} \neq \mathbf{b}_{i}^{\prime}$. This means that the set $A \equiv\left\{t_{i}\right.$ : $\left.\mathbf{b}_{i}\left(t_{i}\right) \neq \mathbf{b}_{i}^{\prime}\left(t_{i}\right)\right\}$ has positive measure. Let $A^{0}$ be the set of types $t_{i}$ in $A$ such that

$$
\operatorname{Pr}\left(\left\{t_{-i}: a_{i}\left(\mathbf{b}_{i}\left(t_{i}\right), \mathbf{b}_{-i}\left(t_{-i}\right)\right) \neq a_{i}\left(\mathbf{b}_{i}^{\prime}\left(t_{i}\right), \mathbf{b}_{-i}\left(t_{-i}\right)\right)\right\}\right)=0,
$$

and let $A^{1} \equiv A \backslash A^{0}$, that is, $A^{1}$ is the set of types $t_{i}$ in $A$ such that the above probability is positive. By Theorem 1 and Remark $1, \operatorname{Pr}\left(A^{1}\right)=0$, which implies that $\operatorname{Pr}(A)=\operatorname{Pr}\left(A^{0}\right)>0$.

Since $a_{i}$ is non-decreasing in $b_{i}$, we have that for every $\alpha \in[0,1]$ and $\overline{\mathbf{b}}_{i}=$ $\alpha \mathbf{b}_{i}+(1-\alpha) \mathbf{b}_{i}^{\prime}$,

$$
\operatorname{Pr}\left(\left\{t_{-i}: a_{i}\left(\overline{\mathbf{b}}_{i}\left(t_{i}\right), \mathbf{b}_{-i}\left(t_{-i}\right)\right) \neq a_{i}\left(\mathbf{b}_{i}^{\prime}\left(t_{i}\right), \mathbf{b}_{-i}\left(t_{-i}\right)\right)\right\}\right)=0,
$$

for all $t_{i} \in A^{0}$. Since $\underline{u}_{i}$ and $\bar{u}_{i}$ are weakly monotonic in the same direction, by Assumption 5 , and $\mathbf{b}_{i}\left(t_{i}\right)$ and $\mathbf{b}_{i}^{\prime}\left(t_{i}\right)$ give the same probability of winning to bidder $i$, it should be that the expectation of $\bar{u}_{i} a_{i}+\underline{u}_{i}\left(1-a_{i}\right)$, that is, the interim payoff, is constant between $\mathbf{b}_{i}\left(t_{i}\right)$ and $\mathbf{b}_{i}^{\prime}\left(t_{i}\right){ }^{17}$ Thus, the convex combination $\overline{\mathbf{b}}_{i}$ gives the same payoff as $\mathbf{b}_{i}$ and $\mathbf{b}_{i}^{\prime}$. Thus, $\overline{\mathbf{b}}_{i} \in \Gamma_{i}\left(\mathbf{b}_{-i}\right)$. It is clear that $\overline{\mathbf{b}}_{i} \in N$, because $\mathbf{b}_{i}, \mathbf{b}_{i}^{\prime} \in N$. This shows that $\Upsilon_{i}$ has convex values.

Define the upper semicontinuous and convex-valued map $\Upsilon: N^{n} \rightarrow N^{n}$ as the product of the $\Upsilon_{i}$. It is defined on the compact convex set $N^{n}$. By Kakutani-FanGlicksberg Theorem, it has a fixed point, denoted by $\mathbf{b}^{m}=\left(\mathbf{b}_{1}^{m}, \ldots, \mathbf{b}_{n}^{m}\right)$, which is an equilibrium of the auction $\mathcal{A}^{m}$.

Proof of Lemma 4. First, we establish the following:

Claim 1. Given that other players are using their strategies $\left(\mathbf{b}_{-i}^{*}, \mathbf{s}_{-i}^{*}\right)$, then for all strategies $\left(\hat{\mathbf{b}}_{i}, \hat{\mathbf{s}}_{i}\right)$ of player $i$ there exists a sequence of strategies $\hat{\mathbf{b}}_{i}^{m}$ such that

$$
\lim _{m \rightarrow \infty} V_{i}^{m}\left(\hat{\mathbf{b}}_{i}^{m}, \mathbf{b}_{-i}^{m}\right)=V_{i}^{*}\left(\left(\hat{\mathbf{b}}_{i}, \hat{\mathbf{s}}_{i}\right),\left(\mathbf{b}_{-i}^{*}, \mathbf{s}_{-i}^{*}\right)\right) .
$$

Proof. If $\hat{\mathbf{s}}_{i}\left(t_{i}\right)$ is such that $\lim _{m} \mathbf{b}_{i}^{m}\left(\hat{\mathbf{s}}_{i}\left(t_{i}\right)\right)=\hat{\mathbf{b}}_{i}\left(t_{i}\right)$, define $\hat{\mathbf{b}}_{i}^{m}\left(t_{i}\right)=\mathbf{b}_{i}^{m}\left(\hat{\mathbf{s}}_{i}\left(t_{i}\right)\right)$. If $\lim \mathbf{b}_{i}^{m}\left(\hat{\mathbf{s}}_{i}\left(t_{i}\right)\right) \neq \hat{\mathbf{b}}_{i}\left(t_{i}\right)$, define $\hat{\mathbf{b}}_{i}^{m}\left(t_{i}\right)=\hat{\mathbf{b}}_{i}\left(t_{i}\right)$ for all $m$. In the first case the limit allocation $a_{i}^{*}\left(\hat{\mathbf{b}}_{i}\left(t_{i}\right), \mathbf{b}_{i}^{*}\left(t_{i}\right), \hat{\mathbf{s}}_{i}, \mathbf{s}_{-i}^{*}\right)$ is

$$
\lim _{m \rightarrow \infty} a_{i}^{m}\left(\mathbf{b}_{i}^{m}\left(\hat{\mathbf{s}}_{i}\left(t_{i}\right)\right), \mathbf{b}_{-i}^{m}\left(t_{-i}\right)\right)=\lim _{m \rightarrow \infty} a_{i}^{m}\left(\hat{\mathbf{b}}_{i}^{m}\left(t_{i}\right), \mathbf{b}_{-i}^{*}\left(t_{-i}\right)\right) .
$$

\footnotetext{
${ }^{17}$ The same argument works if Assumption 5 is changed to the requirement that one of the functions $\underline{u}_{i}$ or $\bar{u}_{i}$ does not depend on $b_{i}$ and the other is quasiconcave as a function of $b_{i}$.
} 
In the second case we have $a_{i}^{m}\left(\hat{\mathbf{b}}_{i}^{m}\left(t_{i}\right), \mathbf{b}_{-i}^{*}\left(t_{-i}\right)\right) \rightarrow a_{i}^{*}\left(\hat{\mathbf{b}}_{i}\left(t_{i}\right), \mathbf{b}_{i}^{*}\left(t_{i}\right), \hat{\mathbf{s}}_{i}, \mathbf{s}_{-i}^{*}\right)$. In both cases we have $\hat{\mathbf{b}}_{i}^{m}\left(t_{i}\right) \rightarrow \hat{\mathbf{b}}_{i}\left(t_{i}\right)$ and

$$
\lim _{m \rightarrow \infty} a_{i}^{m}\left(\hat{\mathbf{b}}_{i}^{m}\left(t_{i}\right), \mathbf{b}_{-i}^{*}\left(t_{-i}\right)\right)=a_{i}^{*}\left(\hat{\mathbf{b}}_{i}\left(t_{i}\right), \mathbf{b}_{i}^{*}\left(t_{i}\right), \hat{\mathbf{s}}_{i}, \mathbf{s}_{-i}^{*}\right) .
$$

Thus, the function $t \mapsto \bar{u}_{i} a_{i}^{m}+\left(1-a_{i}^{m}\right) \underline{u}_{i}$, with bids defined by $\left(\hat{\mathbf{b}}_{i}^{m}, \mathbf{b}_{-i}^{m}\right)$, converge a.e. to $t \mapsto \bar{u}_{i} a_{i}^{*}+\left(1-a_{i}^{*}\right) \underline{u}_{i}$, where the bids and allocations are defined by $\left(\left(\hat{\mathbf{b}}_{i}, \hat{\mathbf{s}}_{i}\right),\left(\mathbf{b}_{-i}^{*}, \mathbf{s}_{-i}^{*}\right)\right)$. Thus, by the Lebesgue convergence theorem, $V_{i}^{m}\left(\hat{\mathbf{b}}_{i}^{m}, \mathbf{b}_{-i}^{m}\right) \rightarrow$ $V_{i}^{*}\left(\left(\hat{\mathbf{b}}_{i}, \hat{\mathbf{s}}_{i}\right),\left(\mathbf{b}_{-i}^{*}, \mathbf{s}_{-i}^{*}\right)\right)$.

The rest of the proof is trivial. Assume that a player $i$ has an improving deviation $\left(\hat{\mathbf{b}}_{i}, \hat{\mathbf{s}}_{i}\right)$ from $\left(\mathbf{b}_{i}^{*}, \mathbf{s}_{i}^{*}\right)$ when the opponents follow $\left(\mathbf{b}_{-i}^{*}, \mathbf{s}_{-i}^{*}\right)$, that is,

$$
V_{i}^{*}\left(\left(\hat{\mathbf{b}}_{i}, \hat{\mathbf{s}}_{i}\right),\left(\mathbf{b}_{-i}^{*}, \mathbf{s}_{-i}^{*}\right)\right)>V_{i}^{*}\left(\left(\mathbf{b}_{i}^{*}, \mathbf{s}_{i}^{*}\right),\left(\mathbf{b}_{-i}^{*}, \mathbf{s}_{-i}^{*}\right)\right)+2 \varepsilon,
$$

for some $\varepsilon>0$. By the claim, there exists a sequence $\hat{\mathbf{b}}_{i}^{m}$ such that for a sufficiently high $m$,

$$
V_{i}^{m}\left(\hat{\mathbf{b}}_{i}^{m}, \mathbf{b}_{-i}^{m}\right)>V_{i}^{m}\left(\mathbf{b}_{i}^{m}, \mathbf{b}_{-i}^{m}\right)+\varepsilon,
$$

which contradicts the fact that $\left(\mathbf{b}_{i}^{m}, \mathbf{b}_{-i}^{m}\right)$ is equilibrium for the auction $\mathcal{A}^{m}$.

Proof of Lemma 5. Consider the first case, that is, there is probability $\gamma>0$ of bids by sellers below $\underline{w}+2 \delta$. Let $\rho^{x}$ the probability of buyer bids above $\underline{w}+2 \delta$. If $\rho^{x} \geq \rho$ for all $x$ and some $\rho>0$, then the probability of trade is at least $\gamma \rho>0$. If $\rho^{x} \rightarrow 0$, then for sufficiently high $x$, there is a probability $1 / 2$ that there is no buy bid above $\underline{w}+2 \delta$. Consider the strategy of buyer $i$ that speficies the bid $b_{O U T}^{B}$ for all types $t_{i}^{\prime}<t_{i}$ and the bid $\underline{w}+2 \delta$ otherwise. From the fact that $p_{i}\left(\underline{w}+2 \delta, \mathbf{b}_{-i}\left(t_{-i}\right)\right) \leq \underline{w}+2 \delta$ (Assumption 7 ) and $\underline{w}+2 \delta<\underline{w}_{i}\left(t_{i}\right)$ (by definition of $\delta$ ), there exists $\eta>0$ such that $U_{i}\left(v_{i}\left(t_{i}^{\prime}, t_{-i}\right)-p_{i}\left(\underline{w}+2 \delta, \mathbf{b}_{-i}\left(t_{-i}\right)\right)\right) \geq \eta$ for all $t_{i}^{\prime} \geq t_{i}$ and $t_{-i} \in[0,1]^{n-1}$. Thus, such strategy ensures at least the payoff $\eta \gamma / 2>0$ for sufficiently high $x$. Thus, it is impossible that trade goes to zero, since this would imply payoff going to zero. By an analogous argument, one obtains a contradiction for the case where there is probability $\gamma>0$ of bids by buyers above $\bar{w}-2 \delta$.

Proof of Lemma 6 . In the expressions below, we will omit the term $\mathbf{b}_{-i}$. The payoff of bidding $\beta_{k}$ is

$$
\begin{aligned}
\Pi_{i}\left(t_{i}, \beta_{k}\right)= & E_{-i}\left[U_{i}\left(v_{i}\left(t_{i}, t_{-i}\right)-p_{i}\left(\beta_{k}, \cdot\right)-e_{i}\right) a_{i}\left(\beta_{k}, \cdot\right)\right] \\
& +E_{-i}\left[U_{i}\left(-e_{i}\right)\left(1-a_{i}\left(\beta_{k}, \cdot\right)\right)\right],
\end{aligned}
$$

for $k=1,2$. The difference $\Pi_{i}\left(t_{i}, \beta_{2}\right)-\Pi_{i}\left(t_{i}, \beta_{1}\right)$ is equal to

$$
\begin{aligned}
& E_{-i}\left\{\left[U_{i}\left(v_{i}\left(t_{i}, t_{-i}\right)-p_{i}\left(\beta_{2}, \cdot\right)-e_{i}\right)-U_{i}\left(-e_{i}\right)\right] a_{i}\left(\beta_{2}, \cdot\right)\right\} \\
& -E_{-i}\left\{\left[U_{i}\left(v_{i}\left(t_{i}, t_{-i}\right)-p_{i}\left(\beta_{1}, \cdot\right)-e_{i}\right)-U_{i}\left(-e_{i}\right)\right] a_{i}\left(\beta_{1}, \cdot\right)\right\} \\
& =E_{-i}\left\{\left[U_{i}\left(v_{i}\left(t_{i}, t_{-i}\right)-p_{i}\left(\beta_{2}, \cdot\right)-e_{i}\right)-U_{i}\left(-e_{i}\right)\right]\left[a_{i}\left(\beta_{2}, \cdot\right)-a_{i}\left(\beta_{1}, \cdot\right)\right]\right\} \\
& -E_{-i}\left\{\left[U_{i}\left(v_{i}\left(t_{i}, t_{-i}\right)-p_{i}\left(\beta_{1}, \cdot\right)-e_{i}\right)-U_{i}\left(v_{i}\left(t_{i}, t_{-i}\right)-p_{i}\left(\beta_{2}, \cdot\right)-e_{i}\right)\right] a_{i}\left(\beta_{1}, \cdot\right)\right\} .
\end{aligned}
$$


Since $v_{i}\left(t_{i}, t_{-i}\right) \geq \underline{w}_{i}\left(t_{i}\right)$ for all $t_{-i}$ and $p_{i}\left(\beta_{2}, \cdot\right) \leq \beta_{2}$, then the term on the third line above is not lesser than

$$
L^{\prime}\left(\underline{w}_{i}\left(t_{i}\right)-\beta_{2}\right) \operatorname{Pr}\left[a_{i}\left(\beta_{2}, \cdot\right)>a_{i}\left(\beta_{1}, \cdot\right)\right] .
$$

On the other hand, since $U_{i}$ is concave, $U_{i}^{\prime} \leq L$ and $\partial_{b_{i}} p_{i} \leq K$,

$$
\begin{aligned}
& U_{i}\left(v_{i}\left(t_{i}, t_{-i}\right)-p_{i}\left(\beta_{1}, \cdot\right)-e_{i}\right)-U_{i}\left(v_{i}\left(t_{i}, t_{-i}\right)-p_{i}\left(\beta_{2}, \cdot\right)-e_{i}\right) \\
\leq & L\left[p_{i}\left(\beta_{2}, \cdot\right)-p_{i}\left(\beta_{1}, \cdot\right)\right] \\
\leq & L K\left(\beta_{2}-\beta_{1}\right) .
\end{aligned}
$$

Thus, the deviation will be profitable if

$$
L^{\prime}\left(\underline{w}_{i}\left(t_{i}\right)-\beta_{2}\right) \operatorname{Pr}\left[a_{i}\left(\beta_{2}, \cdot\right)>a_{i}\left(\beta_{1}, \cdot\right)\right]>L K\left(\beta_{2}-\beta_{1}\right) \operatorname{Pr}\left[a_{i}\left(\beta_{1}, \cdot\right)>0\right] .
$$

Proof of Lemma 7 . The difference $\Pi_{j}\left(t_{j}, \beta_{2}\right)-\Pi_{j}\left(t_{j}, \beta_{1}\right)$ is equal to

$$
\begin{aligned}
& E_{-i}\left[U_{j}\left(v_{j}\left(t_{j}, t_{-j}\right)-e_{i}\right) a_{i}\left(\beta_{2}, \cdot\right)+U_{j}\left(p_{j}\left(\beta_{2}, \cdot\right)-e_{i}\right)\left(1-a_{i}\left(\beta_{2}, \cdot\right)\right)\right] \\
& -E_{-i}\left[U_{j}\left(v_{j}\left(t_{j}, t_{-j}\right)-e_{i}\right) a_{i}\left(\beta_{1}, \cdot\right)+U_{j}\left(p_{j}\left(\beta_{1}, \cdot\right)-e_{i}\right)\left(1-a_{i}\left(\beta_{1}, \cdot\right)\right)\right] \\
= & E_{-i}\left\{\left[U_{j}\left(p_{j}\left(\beta_{2}, \cdot\right)-e_{i}\right)-U_{j}\left(v_{j}\left(t_{j}, t_{-j}\right)-e_{i}\right)\right]\left(a_{i}\left(\beta_{1}, \cdot\right)-a_{i}\left(\beta_{2}, \cdot\right)\right)\right\} \\
& -E_{-i}\left\{\left[U_{j}\left(p_{j}\left(\beta_{1}, \cdot\right)-e_{i}\right)-U_{j}\left(p_{j}\left(\beta_{2}, \cdot\right)-e_{i}\right)\right]\left(1-a_{i}\left(\beta_{1}, \cdot\right)\right)\right\} .
\end{aligned}
$$

As before, we have:

$$
\begin{aligned}
U_{j}\left(p_{j}\left(\beta_{2}, \cdot\right)-e_{i}\right)-U_{j}\left(v_{j}\left(t_{j}, t_{-j}\right)-e_{i}\right) & \geq L^{\prime}\left[p_{j}\left(\beta_{2}, \cdot\right)-\bar{w}_{j}\left(t_{j}\right)\right] \\
& \geq L^{\prime}\left(\beta_{2}-\bar{w}_{j}\left(t_{j}\right)\right),
\end{aligned}
$$

and

$$
U_{j}\left(p_{j}\left(\beta_{1}, \cdot\right)-e_{i}\right)-U_{j}\left(p_{j}\left(\beta_{2}, \cdot\right)-e_{i}\right) \leq L K\left(\beta_{1}-\beta_{2}\right) .
$$

Thus, the deviation will be profitable if

$$
L^{\prime}\left(\beta_{2}-\bar{w}_{j}\left(t_{j}\right)\right) \operatorname{Pr}\left[a_{i}\left(\beta_{1}, \cdot\right)>a_{i}\left(\beta_{2}, \cdot\right)\right]>L K\left(\beta_{1}-\beta_{2}\right) \operatorname{Pr}\left[a_{i}\left(\beta_{1}, \cdot\right)<1\right] .
$$

Proof of Lemma 8. As said before, this is an adaptation of the proof of Theorem 15 of Jackson and Swinkels (2005), henceforth JS. Thus, we will use their notation and refer to their results when they apply to our case. Let $H \subset B$ be the set of buyers $i$ for whom $\bar{w}_{i}=\bar{w}$. By Assumption 8, there is $\omega>0$ such that for each $i \in H$, there is a probability of at least $\omega$ that there is a seller with value below $\bar{w}-\omega$, and such that for each $i \notin H, i$ is a seller or $\bar{w}_{i}<\bar{w}-\omega$. In particular, $\underline{w}<\bar{w}-\omega$.

Consider an arbitrary $k \in\{3,4, \ldots\}$, and let $\delta<\omega / k$. Fix an equilibrium strategy $\mathbf{b}^{x}$. For $i=1, \ldots, n+1$, let $Q_{B i}^{x}=1$ if bidder $i$ is a buyer and bids above $\bar{w}-2 \delta$ and $Q_{B i}^{x}=0$ otherwise. For each $x$, let

$$
\mu_{x}=\max _{i \in H} \operatorname{Pr}_{x}\left\{Q_{B i}^{x}=1\right\}
$$

be the maximum probability that any buyer (not $n+1)$ makes a bid above $\bar{w}-2 \delta$ in $\mathcal{A}^{x}$ (by the definition of $\omega, \operatorname{Pr}_{x}\left\{Q_{B i}^{x}=1\right\}=0$ for each player in $N \backslash H$ ). Let $i^{x}$ be an associated maximizer of $\mu_{x}$. 
Let $Q_{B}^{x}=\sum_{i=1}^{n+1} Q_{B i}^{x}$ be the random variable giving the number of buyer bids above $\bar{w}-2 \delta$ under $\mathbf{b}^{x}$ (including the bids of player $n+1$ ). Let $Q_{S}^{x}$ be the number of seller bids at or below $\bar{w}-2 \delta$. Observe that this does not include player $i^{x}$ who is a buyer.

Lemma 5 establishes that if the trade goes to zero, then $\operatorname{Pr}_{x}\left\{Q_{B}^{x}>0\right\} \rightarrow 0$ and $\operatorname{Pr}_{x}\left\{Q_{S}^{x}>0\right\} \rightarrow 0$ must hold. Now, an argument of JS shows that

$$
\operatorname{Pr}_{x}\left(Q_{B}^{x}=1 \mid Q_{B}^{x}>0\right) \rightarrow 1 .
$$

Choose a subsequence along which $i^{x}$ is constant. Consider the deviation $\mathbf{d}_{j}$ for sellers $j \in S$, that whenever $\bar{w}_{j}\left(t_{j}\right) \leq \bar{w}-\omega$, and the equilibrium specifies a bid above $\bar{w}-2 \delta, j$ submits $\bar{w}-2 \delta$ instead. We will obtain a contradiction if this is not improving for at least one $j \in S$.

Let $C_{j}^{x}$ denote the event where $a_{i}\left(\mathbf{b}_{j}^{x}\left(t_{i}\right), \mathbf{b}_{j}^{x}\left(t_{-j}\right)\right)<1$ and let $D_{j}^{x}$ denote the event where $a_{i}\left(\mathbf{b}_{j}^{x}\left(t_{i}\right), \mathbf{b}_{j}^{x}\left(t_{-j}\right)\right)>a_{i}\left(\mathbf{d}_{j}\left(t_{i}\right), \mathbf{b}_{j}^{x}\left(t_{-j}\right)\right)$. We have the following Corollary to Lemma 7:

Corollary 1. $\mathbf{d}_{j}$ is a profitable deviation for seller $j$ if

$$
L^{\prime}(\omega-2 \delta) \operatorname{Pr}\left[D_{j}^{x}\right]>2 \delta L K \operatorname{Pr}\left[C_{j}^{x}\right] .
$$

Proof. The deviation specifies the bid $\beta_{2}=\bar{w}-2 \delta$, differently from the equilibrium strategy only when $\bar{w}_{j}\left(t_{j}\right) \leq \bar{w}-\omega$, and the equilibrium specifies a bid $\beta_{1} \in(\bar{w}-2 \delta, \bar{w}]$, where the limitation above for $\beta_{1}$ comes from the fact that the seller will never receive more than $\bar{w}$. Then, $\beta_{2}-\bar{w}_{j}\left(t_{j}\right) \geq \omega-2 \delta$ and $\beta_{1}-\beta_{2} \leq 2 \delta$. The result now follows from Lemma 7.

Consider the following set of events, whose dependence on $x$ will be omitted:

$E_{1}: Q_{B}^{x}>0 . .^{18}$

$E_{2}: Q_{S}^{x}=0$.

$E_{3 j}$ : Player $j$ has not sold her unit and $\bar{w}_{j}\left(t_{j}\right) \leq \bar{w}-\omega$.

$E_{4}: Q_{B}^{x} \leq 1$.

$E_{5}$ : There is a seller $j$ such that $\bar{w}_{j}\left(t_{j}\right) \leq \bar{w}-\omega$.

Note that $\operatorname{Pr}_{x}\left(E_{1}\right)=\hat{\mu}_{x}$. Arguing as JS, we have that for $x$ sufficiently large,

$$
\operatorname{Pr}_{x}\left(E_{1} \cap E_{2} \cap E_{4} \cap E_{5}\right) \geq \frac{\omega}{2} \hat{\mu}_{x} .
$$

Since $D_{j}^{x}$ includes the set $E_{1} \cap E_{2} \cap E_{3 j}$, we have

$$
\begin{aligned}
\operatorname{Pr}\left[D_{j}^{x}\right] & \geq \operatorname{Pr}_{x}\left(E_{1} \cap E_{2} \cap E_{3 j} \cap E_{4} \cap E_{5}\right) \\
& \geq \operatorname{Pr}_{x}\left(E_{3 j} \mid E_{1} \cap E_{2} \cap E_{4} \cap E_{5}\right) \frac{\omega}{2} \hat{\mu}_{x} .
\end{aligned}
$$

On the other hand, $j$ sells under $\mathbf{b}^{x}$ with probability $\operatorname{Pr}\left[C_{j}^{x}\right]$, which is bounded by $\operatorname{Pr}_{x}\left(Q_{B}^{x}>0\right)$. JS establishes that $\operatorname{Pr}_{x}\left(Q_{B}^{x}>0\right) \leq n \hat{\mu}_{x}$. From the previous corollary,

\footnotetext{
18 Our definition of this set can be simpler than JS' because in our setting, players are always buyers or always sellers, JS's unlike. This difference is also the reason why we are able to prove the existence of trade when there is just one buyer and one seller, which is not allowed by JS's assumptions.
} 
in order for the deviation not to be profitable, it must be that

$$
L^{\prime}(\omega-2 \delta) \operatorname{Pr}_{x}\left(E_{3 j} \mid E_{1} \cap E_{2} \cap E_{4} \cap E_{5}\right) \frac{\omega}{2} \hat{\mu}_{x} \leq 2 \delta L K n \hat{\mu}_{x} .
$$

Dividing both sides by $\hat{\mu}_{x} \delta$ (which is valid, because $\hat{\mu}_{x}$ is positive thanks to player $n+1)$, and summing across sellers,

$$
\frac{\omega}{2}\left(\frac{\omega}{\delta}-2\right) \sum_{j \in S} \operatorname{Pr}_{x}\left(E_{3 j} \mid E_{1} \cap E_{2} \cap E_{4} \cap E_{5}\right) \leq n_{S} n 2 L K .
$$

But in any realization where $E_{1} \cap E_{2} \cap E_{4} \cap E_{5}$ holds, $E_{3 j}$ must hold for at least one seller $j \in S$, since $E_{5}$ specifies that for at least one seller $j, \bar{w}_{j}\left(t_{j}\right) \leq \bar{w}-\omega$, since no sell bid is at or below $\bar{w}-2 \delta$, and since there is one buy offer above $\bar{w}-2 \delta$. Thus the sum of probabilities above is at least 1 and so

$$
\frac{\omega}{2}\left(\frac{\omega}{\delta}-2\right) \leq n_{S} n 2 L K \text {. }
$$

Recall that $k \in\{3,4, \ldots\}$ is arbitrary and that $\delta$ was chosen so that $\omega / k>\delta$. It follows that

$$
\frac{\omega}{2}(k-2) \leq n_{S} n 2 L K .
$$

This equation is clearly false for $k$ sufficiently large, and we have the desired contradiction.

\section{REFERENCES}

[1] Akerlof, G. A. (1970): "The Market for 'Lemons': Quality Uncertainty and the Market Mechanism", Quarterly Journal of Economics, 84, 488-500.

[2] Amann, E. And W. Leininger (1996): "Asymmetric All-Pay Auctions with Incomplete Information: The Two-Player Case," Games and Economic Behavior, 14, 1996, pp.1-18.

[3] Araujo, A., L. I. de Castro and H. Moreira (2004): "Pure Strategy Equilibria of Multidimensional and Non-Monotonic Auctions," working paper, IMPA.

[4] Athey, S. (2001): "Single Crossing Properties and The Existence of Pure Strategy Equilibria in Games of Incomplete Information," Econometrica, 69, 861-899.

[5] De Castro, L. I. And A. Riascos (2006): "First-Order Condition for Multidimensional Auctions", working paper.

[6] Dunford, N. And Schwartz, J. B. (1958): "Linear Operators. Part I: General Theory." John Wiley \& Sons.

[7] Fudenberg, D., M. M. Mobius And A. Szeidl (2003): "Existence of Equilibrium in Large Double Auctions," forthcoming, Journal of Economic Theory.

[8] Griesmer, J. H., Levitan, R. E and Shubik, M. (1967): "Toward a Study of Bidding Process. Part IV - Games with Unknown Costs," Naval Research Logistics Quarterly, 14, 415-433.

[9] Jackson, M. O., L. K. Simon, J. M. Swinkels and W. R. Zame (2002): "Communication and Equilibrium in Discontinuous Games of Incomplete Information," Econometrica, 70, 17111741.

[10] Jackson, M. O., L. K. Simon, J. M. Swinkels and W. R. Zame (2004), "Corrigendum to 'Communication and Equilibrium in Discontinuous Games of Incomplete Information"', Econometrica, 72, 1927-1929.

[11] Jackson, M. O. and J. M. Swinkels (2005): "Existence of Equilibrium in Single and Double Private Value Auctions," Econometrica, 73, 93-140.

[12] Kallenberg, O. (2002): Foundations of Modern Probability. Springer, New York. 
[13] Lebrun, B. (1999): "First-Price Auction in the Asymmetric N Bidder Case," International Economic Review, 40, 125-142.

[14] Lizzeri, A. And N. Persico (2000): "Uniqueness and Existence of Equilibrium in Auctions with a Reserve Price," Games and Economic Behavior, 30, 83-114.

[15] Maskin, E. And J. Riley (2000): "Equilibrium in Sealed High Bid Auctions," Review of Economic Studies, 67, 439-454.

[16] McAdams, D. (2003): "Isotone Equilibrium in Games of Incomplete Information," Econometrica, 71, 1191-1214.

[17] Milgrom, P. A. (1981): "Rational Expectations, Information Acquisition, and Competitive Bidding," Econometrica, 49, 921-943.

[18] Milgrom, P. A. And C. Shannon (1994): "Monotone Comparative Statics," Econometrica, 62, 157-180.

[19] Reny, P. And Perry, M. (2003): "Toward a Strategic Foundation for Rational Expectations Equilibrium," working paper.

[20] Reny, P. and S. Zamir (2004): "On the Existence of Pure Strategy Monotone Equilibria in Asymmetric First-Price Auctions," Econometrica, 72, 1105-1125.

[21] Simon, L. K. And W. R. Zame (1990): "Discontinuous Games and Endogenous Sharing Rules", Econometrica, 58, 861-872.

[22] Topkis, D. (1978): "Minimizing a Submodular Function on a Lattice," Operations Research, $26,305-321$.

[23] Vickrey, W. (1961): "Counterspeculation, Auctions, and Competitive Sealed Tenders," The Journal of Finance, 16, p. 8-37.

[24] Vives, X. (1961): "Nash Equilibrium with Strategic Complementarities," Journal of Mathematical Economics, 19, p. 305-321.

[25] Williams, S. R. (1991): "Existence and convergence of equilibria in the buyer's bid double auction," Review of Economic Studies, 58, 351-374.

Getulio Vargas Foundation and IMPA

Universidad Carlos III de Madrid 